\title{
Wave Interaction with Defects in Pressurised Composite Structures
}

\author{
R. K. Apalowo ${ }^{1}$ - D. Chronopoulos ${ }^{1} \cdot$ G. Tanner ${ }^{2}$
}

Received: 23 January 2017 / Accepted: 13 June 2018 / Published online: 20 June 2018

(c) The Author(s) 2018

\begin{abstract}
There exists a great variety of structural failure modes which must be frequently inspected to ensure continuous structural integrity of composite structures. This work presents a finite element (FE) based method for calculating wave interaction with damage within structures of arbitrary layering and geometric complexity. The principal novelty is the investigation of pre-stress effect on wave propagation and scattering in layered structures. A wave finite element (WFE) method, which combines FE analysis with periodic structure theory (PST), is used to predict the wave propagation properties along periodic waveguides of the structural system. This is then coupled to the full FE model of a coupling joint within which structural damage is modelled, in order to quantify wave interaction coefficients through the joint. Pre-stress impact is quantified by comparison of results under pressurised and non-pressurised scenarios. The results show that including these pressurisation effects in calculations is essential. This is of specific relevance to aircraft structures being intensely pressurised while on air. Numerical case studies are exhibited for different forms of damage type. The exhibited results are validated against available analytical and experimental results.
\end{abstract}

Keywords Composite structures · Pressurisation · Wave finite element · Wave interaction with damage · Wave propagation

\section{Nomenclature}

$+,-$

a

c

D, $\mathbb{D}$

$\mathbf{k}$

$\mathbf{K}, \mathbf{M}, \mathbf{C} \quad$ Stiffness, mass and damping matrices of a waveguide's modelled periodic segment

q, f Physical displacement and forcing vectors for an elastic waveguide

S Wave scattering matrix

T Wave propagation transfer matrix

z Physical displacement vector for the coupling joint

$\boldsymbol{\phi}, \boldsymbol{\Phi} \quad$ Eigenvector and grouped eigenvector

T Matrix transpose

R. K. Apalowo

eaxrka@nottingham.ac.uk

1 Institute for Aerospace Technology \& The Composites Research Group, The University of Nottingham, NG7 2RD Nottingham, UK

2 School of Mathematical Sciences, University of Nottingham, University Park, NG7 2RD Nottingham, UK $\lambda$

i, n

l)

$\mathbb{K}, \mathbb{M}, \mathbb{C}$

$\omega$

$\Re$

$b, h$

$E, G, \nu, \rho$

$g$

$j$

$k, n, N$

$L$

$L, R, I \quad$ Left, right sides and interior indices

$q, \mathrm{f}$

s

$t$

$w, W$

$x, y, z$
Propagation constant and eigenvalue of the wave propagation eigenproblem

Property of interface and non-interface nodes Property of a coupling joint

Stiffness, mass and damping stiffness matrices of the coupling joint

Angular frequency

Real operator

Width and depth of a cross-section

Elastic modulus, shear modulus, Poisson's ratio and density of an elastic waveguide

Global coordinate index

Number of DoFs on each cross-section of the periodic waveguide segment

Waveguide indices and total number of waveguides existing in the considered system

Length

Displacement and forcing indices

Periodic segment positioning index

Time

Wave eigenvector index and total number of waves accounted for in the waveguide

Property in the $x, y$, or $z$ direction 


\section{Introduction}

Composite structures are increasingly used in modern aerospace and automobile industries due to their well-known benefits. However, they exhibit a wide range of structural failure modes, which include delamination, notch, crack, fibre breakage and fibre-matrix debonding [1], for which the structures have to be frequently and thoroughly inspected in order to ensure continuous structural integrity. Approximately, $27 \%$ of an average modern aircraft's lifecycle cost [2] is dedicated on inspection and repair. The use of 'offline' structural inspection techniques currently leads to a massive reduction of the aircraft's availability and significant financial losses for the operator. Structural health monitoring (SHM) combines non-destructive evaluation (NDE) technologies with new modelling methodologies and robust sensing technologies to detect, identify and monitor the integrity of structures and predict their remaining lifetime. The nondestructive detection and evaluation of damage in industrial structural components during service, is of pertinent importance for monitoring their condition and estimating residual life. This evaluation has been widely studied using the ultrasonic guided wave techniques. These techniques are more sensitive to gross defects compared to micro damage. However, acousto-ultrasonic techniques [3,4], which are excellent for both forms of defects, have been receiving increasing attention during the last decade.

Non-destructive ultrasonic wave distortion during propagation in structural media has been studied as early as in [5]. It has been demonstrated that ultrasonic waves can be successfully employed in non-destructive detection of structural defects and deterioration (such as fatigue) [6-8]. The developed NDE approaches can be classified into matrix formulation techniques: in which ultrasonic waves in layered media are defined by coupling the matrix formulation of each of the layers which constitute the media, and wave propagation techniques: which strongly rely on the calculation of dispersion curves and wave interaction reflection and transmission coefficients to inspect and evaluate structural media. The wave propagation NDE inspection techniques can furthermore be categorised into two steps, namely response and modal steps [9]. The former measures the wave reflection and transmission characteristics of the structure, while the latter determines the wave dispersion and propagation characteristics, such as the wave phase and group velocities as well as the wavenumber. These techniques have been successfully demonstrated in various structural media such as truss [10,11], beams [12], 3-D solid media [13] and composite structure [14]. It has also been applied to calculate wave interaction coefficients from structural joints such as curved [15], spring-type [16], welded [2], adhesive [17], angled [18] and liquid-coupled joints [19].
Implementing a suitable modelling technique is as important as selecting an appropriate NDE method for SHM. The finite element (FE) method [20] is one of the most common ones employed to analyse the dynamic behaviour of structures. The structure is split into a number of elements to form a mesh and equilibrium relationships which are applied to relate the entire structure and boundary conditions to arrive at a unique solution for a specific problem. Finite element based wave propagation NDE technique for periodic structures was first introduced in [21]. It was shown that the wave dispersion characteristics within the layered media can be accurately predicted for a wide frequency range by solving an eigenvalue problem for the wave propagation constants. The work was extended to 2-D media in [22]. The wave finite element (WFE) method was introduced in [23] to facilitate the post-processing of the eigenvalue problem solutions and the improvement of the computational efficiency of the method was presented in [24]. The method is considered as an expansion of Bloch's theorem and its main assumption being the periodicity of the structure to be modelled. It couples the periodic structure theory to the FE method by modelling only a small periodic segment of the structure, thereby saving a whole lot of computational cost and time. WFE method has been successfully implemented in 1-D [23,25] and 2-D [26,27] wave propagation analyses. The method has recently found applications in predicting the vibroacoustic and dynamic performance of layered structures [28]. The variability of acoustic transmission through layered structures [29,30], as well as structural identification [31] have been modelled through the same methodology. The same FE based approach was employed to compute the reflection and transmission coefficients of waves impinging on linear joints in $[25,32]$.

The principal contribution of the work hereby presented is to investigate wave propagation and interaction with defects in periodic structures, and examine the effect of pre-stressing on the wave interaction coefficients. The structure can be of arbitrary complexity, layering and material characteristics as an FE discretisation is employed. The defective structure is discretised into a number of healthy waveguides coupled through a defective coupling joint. Free wave propagation properties of the periodic waveguides are computed through a wave finite element method. A hybrid WFE-FE methodology is then developed to quantify interaction of the WFE computed waves with defect within the full FE defined coupling joint. In general, the structure is pre-stressed by subjecting it to a uniformly distributed surface pressure. The pre-stress effect is evaluated by comparing the wave response (dispersion and reflection properties) of the pressurised structure to that of non-pressurised structure. This is exhibited through presented numerical case studies. 


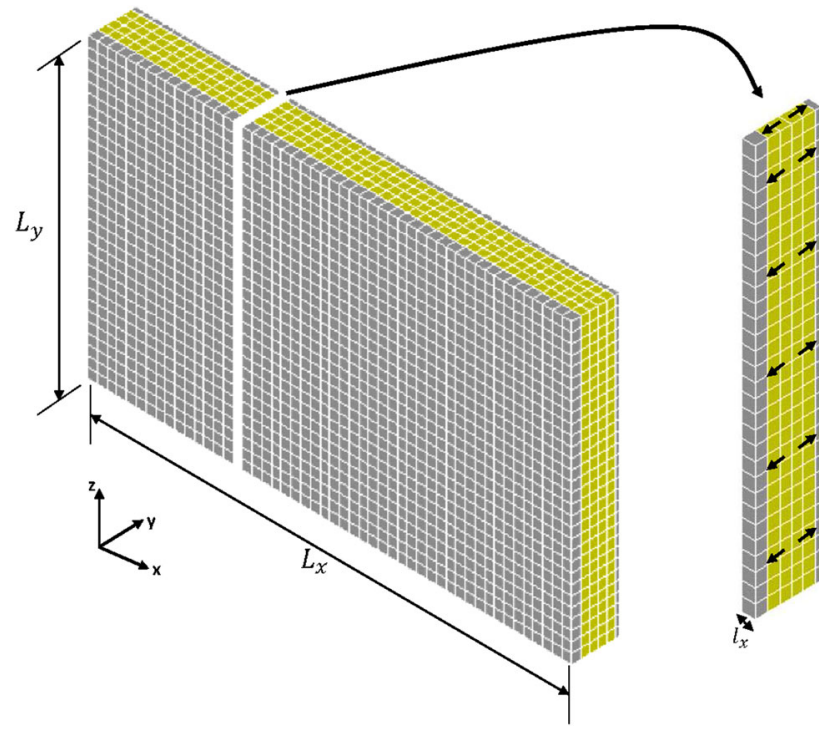

Fig. 1 Internal (core) pressurisation of a periodic segment of a sandwich structure

\section{Stiffness Property of a Pressurised Structure}

Pre-stress impact (due to pressurisation) on the coefficients of wave interaction with defects is examined in this work. Consider an arbitrary periodic segment internally pressurised as shown in Fig. 1. The stress stiffening effect as a result of the applied pressure is accounted for by adding a pre-stress stiffness matrix, $\mathbf{K}_{\mathbf{p}}$, to the unstressed stiffness matrix, $\mathbf{K}_{\mathbf{0}}$, of the system.

$\mathbf{K}_{\mathbf{p}}$ is dependent on the geometry, displacement field and the state of stress of each structural element [33]. For a 3$\mathrm{D}$ element, which is considered in this work, the pre-stress stiffness matrix is given as [34]

$\mathbf{K}_{\mathbf{p}}=\iiint \mathbf{S}_{\mathbf{g}}^{\top} \mathbf{S}_{\mathbf{m}} \mathbf{S}_{\mathbf{g}} \mathrm{d} x \mathrm{~d} y \mathrm{~d} z$

where $\mathbf{S}_{\mathbf{g}}$ is the shape function derivative matrix, $\mathbf{S}_{\mathbf{m}}$ is the Cauchy stress tensor and $[\bullet]^{\top}$ is a transpose. Hence, the total stiffness matrix of the pre-stressed system is given as

$\mathbf{K}=\mathbf{K}_{\mathbf{0}}+\mathbf{K}_{\mathbf{p}}$

Evidently, $\mathbf{K}$ equals to $\mathbf{K}_{\mathbf{0}}$ under no pressurisation scenario.

\section{Finite Element Modelling of Structural Damage}

A system of $\mathrm{N}$ waveguides connected through a coupling joint (Fig. 4) is considered in this study. In the general case, waves travel from one of the waveguides to other waveguides through the joint. Scattering coefficients are calculated from interaction of the waves with structural inconsistencies (such as damage). Composite structures are prone to a number of structural failure modes which range from microscopic fibre faults to large, gross impact damage. Among these failure modes, notch, cracks, delamination and fibre breakage are important modes of failures commonly found in composites $[1,35]$.

Simplified FE methods can be used to simulate the effect of the damage on the mechanical behaviour of the coupling joint. Some of these methods include element deletion, stiffness reduction, duplicate node and kinematics based methods. Descriptions of each of these methods and their applicability are given in the following sections.

\subsection{Element Deletion Method}

This method is mainly applicable for modelling notches such as holes (fibre fractures) and rectangular notches in composites. Here, an element or a number of elements along the axis of the defect is/are deleted from the structure to simulate the effect of the defect. This leads to a reduction in the overall mass and stiffness of the structure. It is one of the simplest FE damage modelling methods as it doesn't require mesh modification.

\subsection{Stiffness Reduction Method}

It is a known fact that structural defects contribute to a reduction in the overall stiffness properties of the structural segment. In this method, the stiffness loss is incorporated in the FE modelling of the structure by multiplying the material property of the structure by a reduction factor $\beta$ as

$P=\beta P_{0}, \quad 0<\beta \leq 1$

where $P$ is the reduced material property, $P_{0}$ the original magnitude of the property (which can be elastic modulus, shear modulus or density). $\beta$ being the reduction factor, equals unity for a pristine structure. This method is applicable to model cracks and delamination, but it is limited to wave interaction problem where mode conversion is not expected.

\subsection{Node Duplication Method}

The node duplication method is applicable for modelling various damage types such as single and multiple delamination and cracks, and fibre breakages.

In this method, nodes along axis of the crack, within the structural segment, are disconnected by adding duplicate nodes, which have the same nodal coordinates but different nodes numbers, to the nodes being disconnected. Each duplicate node is assigned to an adjacent element such 
Fig. 2 Finite element modelling of damage using node duplication method: a damaged structural segment, b node duplication model (a)

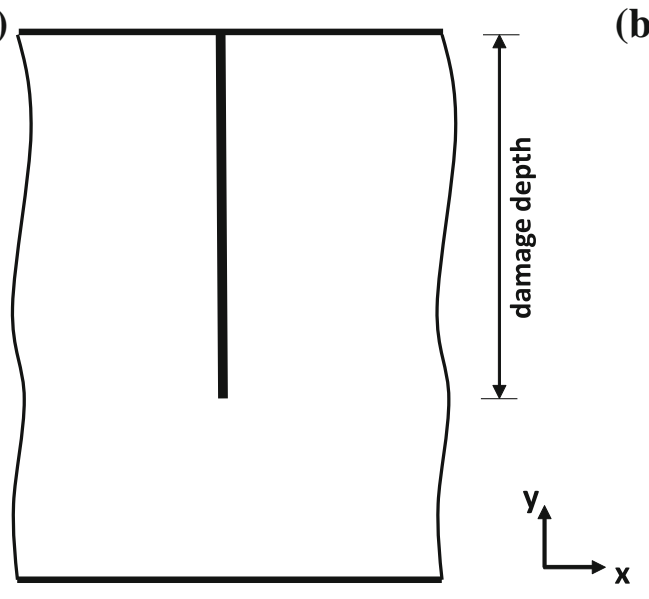

(b)

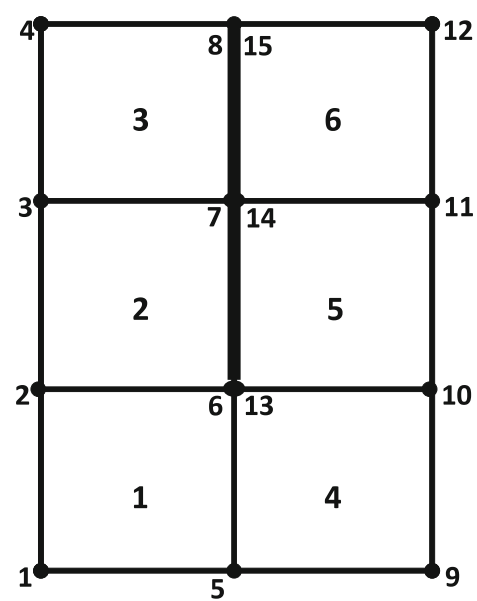

that when a tensile force is applied, the nodes along the crack front are separated. In this respect, if the original nodes are connected to the left side elements, the duplicate nodes will be connected to the elements on the right side.

As an illustration of this method, a structural segment with six plane strain FEs is considered. Elements and nodes numbering of the segment are as shown Fig. 2. For the damage depth considered, nodes 6,7 and 8 , which are along the damage axis, are disconnected by adding duplicate nodes 13,14 and 15 of same respective nodal coordinates. In a pristine state of the segment, nodal arrangement of finite element 2 is $[2,6,7,3]$ in that order, while that of element 5 is $[6,10,11,7]$. But, in a damaged state, nodal arrangement of element 2 remains $[2,6,7,3]$ while that of element 5 becomes $[13,10,11,14]$ to model defects at the interface of the two FEs. Similar node ordering holds for elements 3 and 6 with nodal arrangements $[3,7,8,4]$ and $[14,11$, $12,15]$ respectively in the damaged state of the structural segment.

Although a 2D structural segment is used to illustrate the procedure of this method, extending the procedure to model damage in a 3D structure is quite similar and straightforward.

\subsection{Kinematics Based Method}

This approach has a lot of similarities to the node duplication method. It involves enforcing kinematics to the nodes surrounding the damage. The structural segment is segmented into multiple domains along the crack front. The stiffness and mass matrices of each domain are generated and coupled to obtain the overall matrices of the structural segment. More details on the approach can be found in [36]. The method is applicable to model delamination, cracks and fibre breakages.

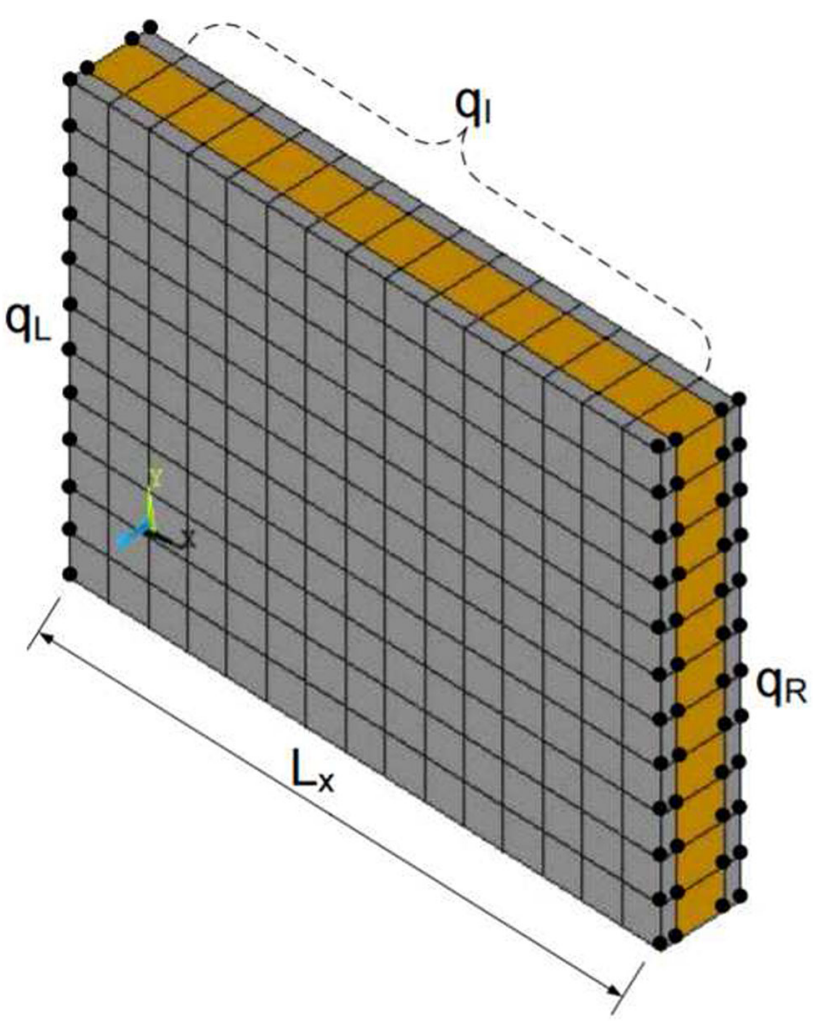

Fig. 3 WFE modelled layered composite waveguide with the left and right side nodes $\mathbf{q}_{\mathbf{L}}, \mathbf{q}_{\mathbf{R}}$ bullet marked. The range of interior nodes $\mathbf{q}_{\mathbf{I}}$ also illustrated

\section{Free Wave Propagation in an Arbitrarily Periodic Structure by WFE Method}

Linear elastic wave propagation is considered in the $x$ direction of the arbitrary periodic structural waveguide of Fig. 3. A FE model, of a periodic segment of the structural waveguide, is meshed using commercial FEA software.

The problem can be condensed using a transfer matrix approach as in [23]. The frequency dependent dynamic stiff- 
ness matrix (DSM), D, which relates the nodal displacements and the internal forces (of the periodic segment's nodes) under a time-harmonic behaviour assumption, is given as

$\left[\mathbf{K}+\mathbf{i} \omega \mathbf{C}-\omega^{2} \mathbf{M}\right] \mathbf{q}=\mathbf{f}$

where $\mathbf{q}$ and $\mathbf{f}$ are the displacement and internal force vectors respectively. $\mathbf{C}$ and $\mathbf{M}$ are respectively the damping and mass matrices of the segment. The internal force vector is responsible for transmitting waves from one element to the other within the structure, hence it is not zero, even for a free wave motion where no external load is applied [27], as is the case being considered in this work.

The DSM can be partitioned with regards to the left $L$, right $R$ and internal $I$ DoFs of the periodic segment as

$$
\left[\begin{array}{lll}
D_{L L} & D_{L I} & D_{L R} \\
D_{I L} & D_{I I} & D_{I R} \\
D_{R L} & D_{R I} & D_{R R}
\end{array}\right]\left\{\begin{array}{c}
q_{L} \\
q_{I} \\
q_{R}
\end{array}\right\}=\left\{\begin{array}{c}
f_{L} \\
f_{I} \\
f_{R}
\end{array}\right\}
$$

Using a dynamic condensation technique for the internal nodes DoFs, Eq. (5) can be expressed in the form

$$
\left[\begin{array}{ll}
D_{L L}-D_{L I} D_{I I}^{-1} D_{I L} & D_{L R}-D_{L I} D_{I I}^{-1} D_{I R} \\
D_{R L}-D_{R I} D_{I I}^{-1} D_{I L} & D_{R R}-D_{R I} D_{I I}^{-1} D_{I R}
\end{array}\right]\left\{\begin{array}{l}
q_{L} \\
q_{R}
\end{array}\right\}=\left\{\begin{array}{c}
f_{L} \\
f_{R}
\end{array}\right\}
$$

As earlier stated, it is assumed that no external forces are applied on the segment. As a result of this, the displacement continuity and equilibrium of forces equations at the interface of two consecutive periodic segments $s$ and $s+1$ are given as

$$
\begin{aligned}
& \mathbf{q}_{\mathbf{R}}^{\mathbf{s}}=\mathbf{q}_{\mathbf{L}}^{\mathbf{s}+\mathbf{1}} \\
& \mathbf{f}_{\mathbf{R}}^{\mathrm{s}}=-\mathbf{f}_{\mathbf{L}}^{\mathrm{s}+\mathbf{1}}
\end{aligned}
$$

The transfer matrix, $\mathbf{T}$, relates the displacement and force vectors of the left and right sides of the periodic segments. This is done by combining Eqs. (6) and (7) as

$$
\left\{\begin{array}{l}
\mathbf{q}_{\mathbf{L}}^{s+1} \\
\mathbf{f}_{\mathbf{L}}^{s+1}
\end{array}\right\}=\mathbf{T}\left\{\begin{array}{l}
\mathbf{q}_{\mathbf{L}}^{\mathbf{s}} \\
\mathbf{f}_{\mathbf{L}}^{s}
\end{array}\right\}
$$

and the expression for the symplectic transfer matrix is defined as

$$
T=\left[\begin{array}{ll}
\mathbf{D}_{11} & \mathbf{D}_{12} \\
\mathbf{D}_{21} & \mathbf{D}_{22}
\end{array}\right]
$$

with

$$
\begin{aligned}
& \mathbf{D}_{11}=-\left(\mathbf{D}_{\mathrm{LR}}-\mathbf{D}_{\mathrm{LI}} \mathbf{D}_{\mathrm{II}}^{-1} \mathbf{D}_{\mathrm{IR}}\right)^{-1}\left(\mathbf{D}_{\mathrm{LL}}-\mathbf{D}_{\mathrm{LI}} \mathbf{D}_{\mathrm{II}}^{-1} \mathbf{D}_{\mathrm{IL}}\right) \\
& \mathbf{D}_{\mathbf{1 2}}=\left(\mathbf{D}_{\mathrm{LR}}-\mathbf{D}_{\mathrm{LI}} \mathbf{D}_{\mathrm{II}}^{-1} \mathbf{D}_{\mathrm{IR}}\right)^{-\mathbf{1}} \\
& \mathbf{D}_{\mathbf{2 1}}=\left(-\mathbf{D}_{\mathbf{R L}}+\mathbf{D}_{\mathbf{R I}} \mathbf{D}_{\mathrm{II}}^{-1} \mathbf{D}_{\mathbf{I L}}\right)+\left(\mathbf{D}_{\mathbf{R R}}+\mathbf{D}_{\mathbf{R I}} \mathbf{D}_{\mathbf{I I}}^{-1} \mathbf{D}_{\mathrm{IR}}\right) \\
& \times\left(\mathbf{D}_{\mathbf{L R}}-\mathbf{D}_{\mathbf{L I}} \mathbf{D}_{\mathrm{II}}^{-1} \mathbf{D}_{\mathrm{IR}}\right)^{-\mathbf{1}}\left(\mathbf{D}_{\mathrm{LL}}-\mathbf{D}_{\mathrm{LI}} \mathbf{D}_{\mathrm{II}}^{-1} \mathbf{D}_{\mathrm{IL}}\right) \\
& \mathbf{D}_{\mathbf{2 2}}=-\left(\mathbf{D}_{\mathbf{R R}}+\mathbf{D}_{\mathbf{R I}} \mathbf{D}_{\mathbf{I I}}^{-\mathbf{1}} \mathbf{D}_{\mathbf{I R}}\right)\left(\mathbf{D}_{\mathbf{L R}}-\mathbf{D}_{\mathbf{L I}} \mathbf{D}_{\mathbf{I I}}^{-\mathbf{1}} \mathbf{D}_{\mathrm{IR}}\right)^{-\mathbf{1}}
\end{aligned}
$$

With a wave propagating freely along the x-direction (1dimensional wave propagation), the propagation constant, $\lambda=e^{-i k L_{x}}$, relates the left and right nodal displacements and forces by

$$
\begin{array}{r}
\mathbf{q}_{\mathbf{R}}^{\mathbf{s}}=\lambda \mathbf{q}_{\mathbf{L}}^{\mathbf{s}} \\
\mathbf{f}_{\mathbf{R}}^{\mathbf{s}}=-\lambda \mathbf{f}_{\mathbf{L}}^{\mathbf{s}}
\end{array}
$$

By substituting Eqs. (7) and (11) in Eq. (8), the free wave propagation is described by the eigenproblem relation

$$
\lambda\left\{\begin{array}{c}
\mathbf{q}_{\mathbf{L}}^{\mathbf{s}} \\
\mathbf{f}_{\mathbf{L}}^{\mathbf{s}}
\end{array}\right\}=\mathbf{T}\left\{\begin{array}{c}
\mathbf{q}_{\mathbf{L}}^{\mathbf{s}} \\
\mathbf{f}_{\mathbf{L}}^{\mathbf{s}}
\end{array}\right\}
$$

whose eigenvalues $\lambda_{\omega}$ and eigenvectors $\boldsymbol{\phi}_{\omega}=\lambda\left\{\begin{array}{l}\boldsymbol{\phi}_{q} \\ \boldsymbol{\phi}_{f}\end{array}\right\}_{\omega}$ solution sets provide a comprehensive description of the propagation constants and the wave mode shapes for each of the elastic waves propagating in the structural waveguide at a specified angular frequency $\omega$. Both positive going (with $\lambda_{\omega}^{+}$and $\boldsymbol{\phi}_{\omega}^{+}$) and negative going (with $\lambda_{\omega}^{-}$and $\boldsymbol{\phi}_{\omega}^{-}$) waves are sought through the eigensolution. Positive going waves are characterised [23] by

$$
\begin{aligned}
& \left|\lambda_{\omega}^{+}\right| \leq 1 \\
& \Re\left(i \omega \boldsymbol{\phi}_{f}^{+\top} \boldsymbol{\phi}_{q}^{+}\right)<0 \text { if }\left|\lambda_{\omega}^{+}\right|=1
\end{aligned}
$$

which states that when a wave is propagating in the positive $x$ direction, its amplitude should be decreasing, or that if its amplitude is constant (in the case of propagating waves with no attenuation), then there is time average power transmission in the positive direction. Then the wavenumbers of the waves (at a specified angular frequency) in the positive $\mathbf{k}_{\omega}^{+}$ and the negative $\mathbf{k}_{\boldsymbol{\omega}}^{-}$directions can be determined from the propagation constants as

$$
\begin{aligned}
& k_{\omega}^{+}=-\frac{\ln \left(\lambda_{\omega}^{+}\right)}{i L_{x}} \\
& k_{\omega}^{-}=-\frac{\ln \left(\lambda_{\omega}^{-}\right)}{i L_{x}}
\end{aligned}
$$


Fig. 4 Periodic elastic waveguides connected through a coupling joint. Waves having amplitudes $\mathbf{a}_{\mathbf{n}}^{+}$impinging on the joint from the $n$th waveguide will give rise to waves of reflection coefficients $\mathbf{c}_{\mathbf{n}, \mathbf{n}}$ in the $n$th waveguide and waves of transmission coefficients $\mathbf{c}_{\mathbf{k}, \mathbf{n}}$ in the $k$ th waveguide

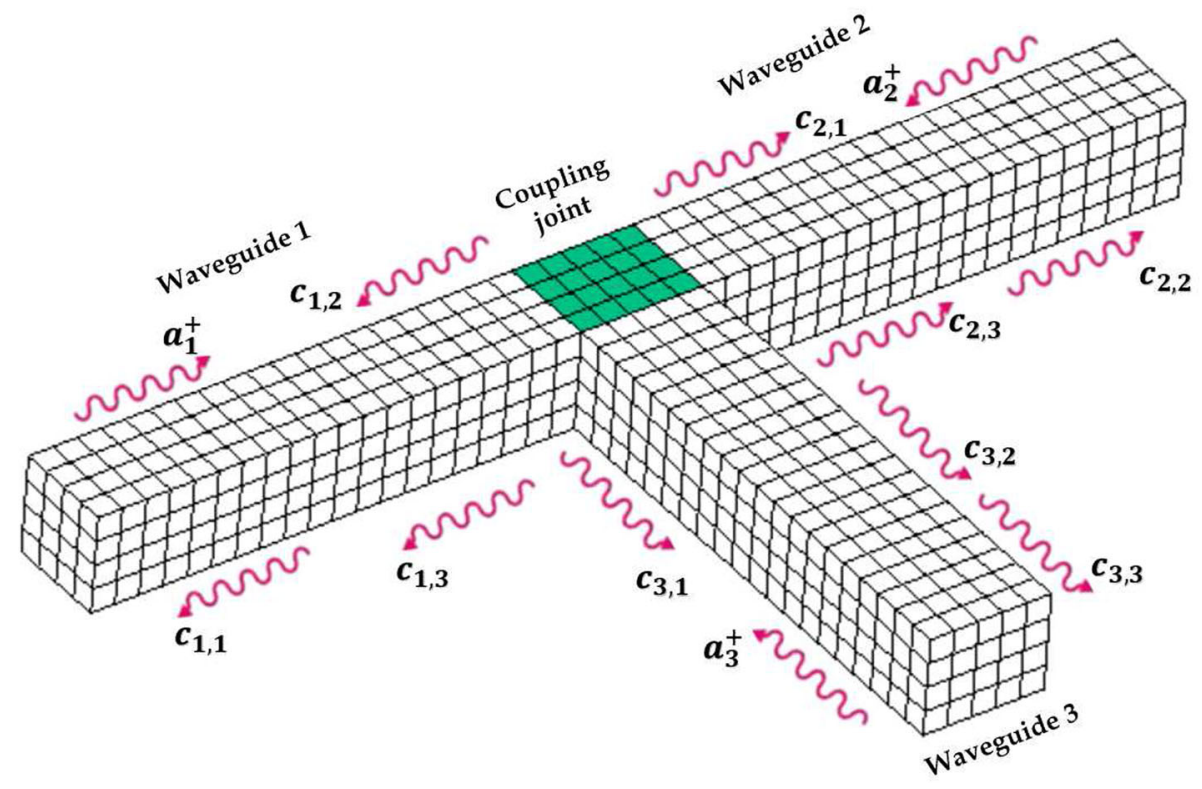

\section{Elastic Wave Interaction Modelling by Hybrid WFE-FE Approach}

In the general case, a system of $N$ healthy periodic waveguides connected through a structural coupling joint as shown in Fig. 4 is considered. The coupling joint could exhibit arbitrarily complex mechanical behaviour such as damage, geometric or material inconsistencies and is fully FE modelled. As already stated, each waveguide can be of different and arbitrary layering and can also support a number $(W)$ of propagating waves at a given frequency.

Propagation constants of the waves are sought through the WFE methodology as presented in Sect. 4. Each supported wavemode $w$ with $w \in[1 \cdots W]$ for waveguide $n$ with $n \in[1 \cdots N]$ in the system can be grouped as

$$
\begin{aligned}
\boldsymbol{\Phi}_{\mathbf{n}, \mathbf{q}}^{+} & =\left[\begin{array}{llll}
\boldsymbol{\phi}_{q, 1}^{+} & \boldsymbol{\phi}_{q, 2}^{+} & \cdots & \boldsymbol{\phi}_{q, W}^{+}
\end{array}\right] \\
\boldsymbol{\Phi}_{\mathbf{n}, \mathbf{f}}^{+} & =\left[\begin{array}{llll}
\boldsymbol{\phi}_{f, 1}^{+} & \boldsymbol{\phi}_{f, 2}^{+} & \cdots & \boldsymbol{\phi}_{f, W}^{+}
\end{array}\right] \\
\boldsymbol{\Phi}_{\mathbf{n}, \mathbf{q}}^{-} & =\left[\begin{array}{llll}
\boldsymbol{\phi}_{q, 1}^{-} & \boldsymbol{\phi}_{q, 2}^{-} & \cdots & \boldsymbol{\phi}_{q, W}^{-}
\end{array}\right] \\
\boldsymbol{\Phi}_{\mathbf{n}, \mathbf{f}}^{-} & =\left[\begin{array}{llll}
\boldsymbol{\phi}_{f, 1}^{-} & \boldsymbol{\phi}_{f, 2}^{-} & \cdots & \boldsymbol{\phi}_{f, W}^{-}
\end{array}\right]
\end{aligned}
$$

with each matrix being of dimension $[j \times W]$. The wavemodes of the entire system can be computed at each specified angular frequency and be grouped as

$$
\boldsymbol{\Phi}_{\boldsymbol{q}}^{+}=\left[\begin{array}{cccc}
\boldsymbol{\Phi}_{1, \mathbf{q}}^{+} & \mathbf{0} & \cdots & \mathbf{0} \\
\mathbf{0} & \boldsymbol{\Phi}_{\mathbf{2}, \mathbf{q}}^{+} & \cdots & \mathbf{0} \\
\cdots & \cdots & \cdots & \cdots \\
\mathbf{0} & \mathbf{0} & \cdots & \boldsymbol{\Phi}_{\mathbf{N}, \mathbf{q}}^{+}
\end{array}\right]_{[j N \times W N]}
$$

with respective similar expressions for $\boldsymbol{\Phi}_{\mathbf{f}}^{+}, \boldsymbol{\Phi}_{\mathbf{q}}^{-}$and $\boldsymbol{\Phi}_{\mathbf{f}}^{-}$. A local coordinate system is defined for each waveguide such that the waveguide's positive axis is directed towards the joint. The rotation matrix $\mathbf{R}_{\mathbf{n}}$ transforms the DoFs of each waveguide from the local to the global coordinates of the system as

$\boldsymbol{\Phi}_{\mathbf{q}}^{\mathbf{g},+}=\mathbf{R} \boldsymbol{\Phi}_{\mathbf{q}}^{+}$

with respective similar expressions for $\boldsymbol{\Phi}_{\mathbf{f}}^{\mathbf{g},+}, \boldsymbol{\Phi}_{\mathbf{q}}^{\mathbf{g},-}$ and $\boldsymbol{\Phi}_{\mathbf{f}}^{\mathbf{g},-}$. $\mathrm{g}$ denotes the global coordinates index and $\mathbf{R}$ represents the rotation matrices of the system's waveguides, grouped in a block diagonal matrix as

$$
\mathbf{R}=\left[\begin{array}{cccc}
\mathbf{R}_{\mathbf{1}} & \mathbf{0} & \cdots & \mathbf{0} \\
\mathbf{0} & \mathbf{R}_{\mathbf{2}} & \cdots & \mathbf{0} \\
\cdots & \cdots & \cdots & \cdots \\
\mathbf{0} & \mathbf{0} & \cdots & \mathbf{R}_{\mathbf{N}}
\end{array}\right]_{[j N \times j N]}
$$

The equation of motion for the FE modelled coupling joint can be in general written as

$M \ddot{z}(\mathbf{t})+\mathbb{C} \dot{\mathbf{z}}(\mathbf{t})+\mathbb{K}_{\mathbf{z}}(\mathbf{t})=\mathbf{f}_{\mathbf{e}}(\mathbf{t})$

with the frequency dependent DSM of the joint expressed as

$\mathbb{D}=\mathbb{K}+i \omega \mathbb{C}-\omega^{2} \mathbb{M}$

where $\mathbb{K}, \mathbb{C}$ and $M$ are stiffness, damping and mass matrices of the coupling joint, $\mathbf{z}$ is the physical displacement vector of the coupling joint and $\mathbf{f}_{\mathbf{e}}$ is the set of elastic forces applied to the coupling joint at its interface with connected waveguides. It is assumed that all connected waveguides are considered 
to be purely elastic and that no external force is applied at the non-interface nodes of the joint. As a result of this and similar to the waveguides, the DSM of the joint can be partitioned with regards to the interface $₫$ and non-interface $\mathrm{m}$ nodes of the joint with the waveguides as

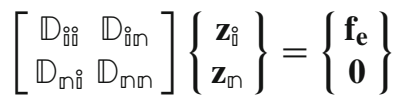

Using a dynamic condensation for the non-interface DoFs, Eq. (21) can be expressed as

$$
\mathbb{D}_{\downarrow} \mathbf{Z}_{\mathfrak{0}}(t)=\mathbf{f}_{\mathbf{e}}(\mathbf{t})
$$

with

$\mathbb{D}_{\jmath}=\mathbb{D}_{\mathfrak{0}}-\mathbb{D}_{\mathfrak{\imath} n} \mathbb{D}_{m n}^{-1} \mathbb{D}_{\text {mi }}$

where $\mathbb{D}_{\jmath}$ is of dimension $[j N \times W N]$.

The equilibrium of forces at the coupling joint gives

$\mathbf{f}_{\mathbf{e}}(\mathbf{t})-\mathbf{R f}(\mathbf{t})=\mathbf{0}$

where $\mathbf{f}$ is the set of forces applied by the waveguides connected to the joint and the continuity conditions for the joint give

$\mathbf{z}_{\mathbf{i}}(\mathbf{t})=\mathbf{R q}(\mathbf{t})$

with

$\left.\mathbf{q}=\left[\begin{array}{llll}\mathbf{q}_{1} & \mathbf{q}_{2} & \cdots & \mathbf{q}_{\mathbf{N}}\end{array}\right]_{[\mathrm{jN} \times \mathbf{1}}^{\top}\right]$

Waves of amplitudes $\mathbf{a}_{\mathbf{n}}^{+}$are impinging on the coupling joint from the $n$th waveguide. These give rise to reflected waves of amplitudes $\mathbf{a}_{\mathbf{n}}^{-}$in the $n$th waveguide and transmitted waves of amplitudes $\mathbf{a}_{\mathbf{k}}^{-}$in the $k$ th waveguide (and vice versa as shown in Fig. 4) expressed as

$\mathbf{a}_{\mathbf{n}}^{-}=\mathbf{c}_{\mathbf{n}, \mathbf{n}} \mathbf{a}_{\mathbf{n}}^{+}$

$\mathbf{a}_{\mathbf{k}}^{-}=\mathbf{c}_{\mathbf{k}, \mathbf{n}} \mathbf{a}_{\mathbf{n}}^{+}$

with $\mathbf{c}_{\mathbf{n}, \mathbf{n}}$ and $\mathbf{c}_{\mathbf{k}, \mathbf{n}}$ respectively being matrices containing the reflection and transmission coefficients of the scattered waves. Hence, the incident waves amplitudes can be related to the amplitudes of the scattered waves as

$\mathbf{a}^{-}=\mathbf{S a}^{+}$

where $\mathbf{a}_{[\mathbf{W N} \times \mathbf{1}]}^{+}$is the vector containing the amplitudes of the incident waves moving towards the coupling joint and $\mathbf{a}_{[\mathbf{W N} \times \mathbf{1}]}^{-}$the vector containing the amplitudes of the reflected and transmitted scattered waves. The wave scattering matrix
$\mathbf{S}$ whose diagonal and off-diagonal elements respectively represent the reflection and transmission coefficients of the scattered waves can be expressed in the form

$\mathbf{S}=\left[\begin{array}{ccccc}\mathbf{c}_{1,1} & \cdots & \mathbf{c}_{1, \mathbf{W}} & \cdots & \mathbf{c}_{\mathbf{1}, \mathbf{W N}} \\ \cdots & \cdots & \cdots & \cdots & \cdots \\ \mathbf{c}_{\mathbf{W}, \mathbf{1}} & \cdots & \mathbf{c}_{\mathbf{W}, \mathbf{W}} & \cdots & \mathbf{c}_{\mathbf{W}, \mathbf{W N}} \\ \cdots & \cdots & \cdots & \cdots & \cdots \\ \mathbf{c}_{\mathbf{W N}, \mathbf{1}} & \cdots & \mathbf{c}_{\mathbf{W N}, \mathbf{W}} & \cdots & \mathbf{c}_{\mathbf{W N}, \mathbf{W N}}\end{array}\right]_{[W N \times W N]}$

A transformation can be defined for the motion in the waveguides between the physical domain, where the motion is described in terms of $\mathbf{q}(\mathbf{t})$ and $\mathbf{f}(\mathbf{t})$ and the wave domain, where the motion is described in terms of waves of amplitudes $\mathbf{a}^{+}$and $\mathbf{a}^{-}$travelling in the positive and negative directions respectively as

$$
\begin{array}{r}
\mathbf{q}_{\mathbf{n}}(\mathbf{t})=\boldsymbol{\Phi}_{\mathbf{n}, \mathbf{q}}^{+} \mathbf{a}_{\mathbf{n}}^{+} \cos (\omega \mathbf{t})+\boldsymbol{\Phi}_{\mathbf{n}, \mathbf{q}}^{-} \mathbf{a}_{\mathbf{n}}^{-} \cos (\omega \mathbf{t}) \\
\mathbf{f}_{\mathbf{n}}(\mathbf{t})=\boldsymbol{\Phi}_{\mathbf{n}, \mathbf{f}}^{+} \mathbf{a}_{\mathbf{n}}^{+} \cos (\omega \mathbf{t})+\boldsymbol{\Phi}_{\mathbf{n}, \mathbf{f}}^{-} \mathbf{a}_{\mathbf{n}}^{-} \cos (\omega \mathbf{t})
\end{array}
$$

and by concatenating the corresponding vectors and matrices, the general expressions for $\mathbf{q}(\mathbf{t})$ and $\mathbf{f}(\mathbf{t})$ for the system's waveguides can be expressed as

$$
\begin{aligned}
& \mathbf{q}(\mathbf{t})=\boldsymbol{\Phi}_{\mathbf{q}}^{+} \mathbf{a}_{\mathbf{n}}^{+} \cos (\omega \mathbf{t})+\boldsymbol{\Phi}_{\mathbf{q}}^{-} \mathbf{a}_{\mathbf{n}}^{-} \cos (\omega \mathbf{t}) \\
& \mathbf{f}(\mathbf{t})=\boldsymbol{\Phi}_{\mathbf{f}}^{+} \mathbf{a}_{\mathbf{n}}^{+} \cos (\omega \mathbf{t})+\boldsymbol{\Phi}_{\mathbf{f}}^{-} \mathbf{a}_{\mathbf{n}}^{-} \cos (\omega \mathbf{t})
\end{aligned}
$$

Substituting Eq. (22) into the equilibrium equation (Eq. (24)) and then substitute the continuity equation (Eq. (25)) into the resulting expression gives

$$
\mathbb{D}_{\jmath} \mathbf{R q}=\mathbf{R f}
$$

Substituting Eqs. (17) and (31) in Eq. (32) and express the resulting equation in the form of Eq. (28) gives the wave interaction scattering matrix as

$\mathbf{S}=-\left[\boldsymbol{\Phi}_{\mathbf{f}}^{\mathrm{g},-}-\mathbb{D}_{\downarrow} \boldsymbol{\Phi}_{\mathbf{q}}^{\mathrm{g},-}\right]^{-1}\left[\Phi_{\mathbf{f}}^{\mathrm{g},+}-\mathbb{D}_{\jmath} \Phi_{\mathbf{q}}^{\mathrm{g},+}\right]$

\section{Numerical Case Studies}

This section presents case studies to demonstrate the application of the developed methodology. The case studies are divided into two; validation and test case studies. The validation cases are presented for models whose analytical and experimental wave dispersion and scattering properties can be obtained. The analytical and the experimental results are compared to the numerically predicted results in order to illustrate the validity of the presented methodology. The test 
Fig. 5 Two bars connected through another bar (coupling joint)

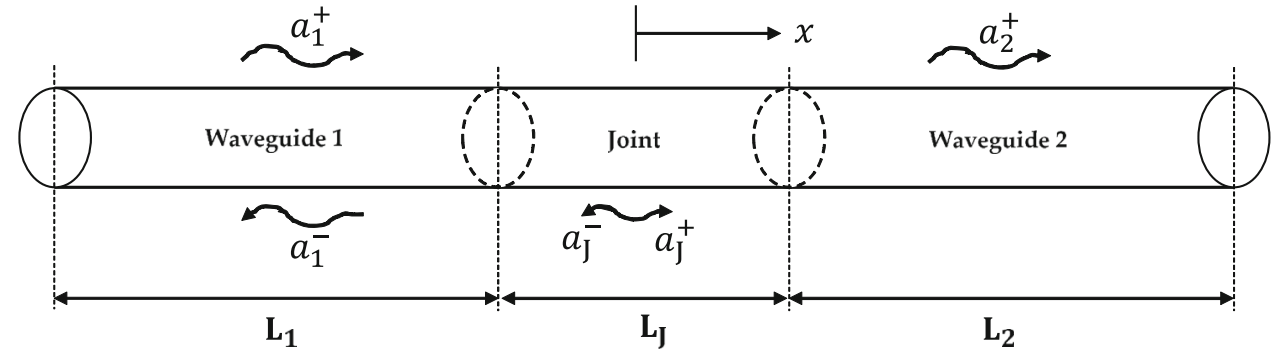

cases present the application of the proposed scheme in computing waves propagation constants and quantifying waves interaction with defects within damaged layered structures subjected to pressurisation. Effect of pre-stress (due to pressurisation) on these waves properties is also examined. In all cases, finite element size is chosen to ensure that mesh density is fine enough to represent the structure accurately at a reasonable computational cost. All properties and dimensions are in SI units, unless otherwise stated.

\subsection{Validation Case Studies}

\subsubsection{Two Collinear Bars Coupled Through a Finite Bar}

Two collinear bars connected through another bar (the coupling joint) of a different material characteristics is considered. The bars are of uniform circular cross-section and undergo longitudinal vibration. Arrangement of the bars is presented in Fig. 5. Each waveguide is made of aluminium $\left(E_{1}=E_{2}=70 \times 10^{9}, \rho_{1}=\rho_{2}=2600\right)$ and the joint is made of steel $\left(E_{J}=210 \times 10^{9}, \rho_{J}=7500\right)$. Cross-sectional areas $A_{1}=A_{2}=A_{J}=0.003$, lengths $L_{1}=L_{2}=0.2$ and $L_{J}=0.003$.

Incident wave of amplitude $\mathbf{a}_{1}^{+}$impinging on the coupling joint from waveguide 1 will give rise to reflected and transmitted waves of amplitudes $\mathbf{a}_{1}^{-}$and $\mathbf{a}_{2}^{+}$in waveguides 1 and 2 respectively. Standing wave is present in the joint since both forward and backward moving waves of amplitudes $\mathbf{a}_{\mathbf{J}}^{+}$and $\mathbf{a}_{\mathbf{J}}^{-}$are simultaneously present.

Imposing necessary boundary conditions across the coupling joint gives the transfer function of the waves as [15]

$[B]\left\{\mathbf{a}_{\mathbf{1}}^{-} \mathbf{a}_{\mathbf{J}}^{+} \mathbf{a}_{\mathbf{J}}^{-} \mathbf{a}_{\mathbf{2}}^{+}\right\}^{\top}=\left\{\begin{array}{lllll}1 & k_{1} E_{1} A_{1} & 0 & 0\end{array}\right\}^{\top} \mathbf{a}_{\mathbf{1}}^{+}$

with

$B=\left[\begin{array}{cccc}-1 & 1 & 1 & 0 \\ k_{1} E_{1} A_{1} & k_{J} E_{J} A_{J} & -k_{J} E_{J} A_{J} & 0 \\ 0 & e^{-i k_{J} L} & e^{i k_{J} L} & -e^{-i k_{2} L} \\ 0 & -k_{J} E_{J} A_{J} e^{-i k_{J} L} & k_{J} E_{J} A_{J} e^{i k_{J} L} & k_{2} E_{2} A_{2} e^{-i k_{2} L}\end{array}\right]$

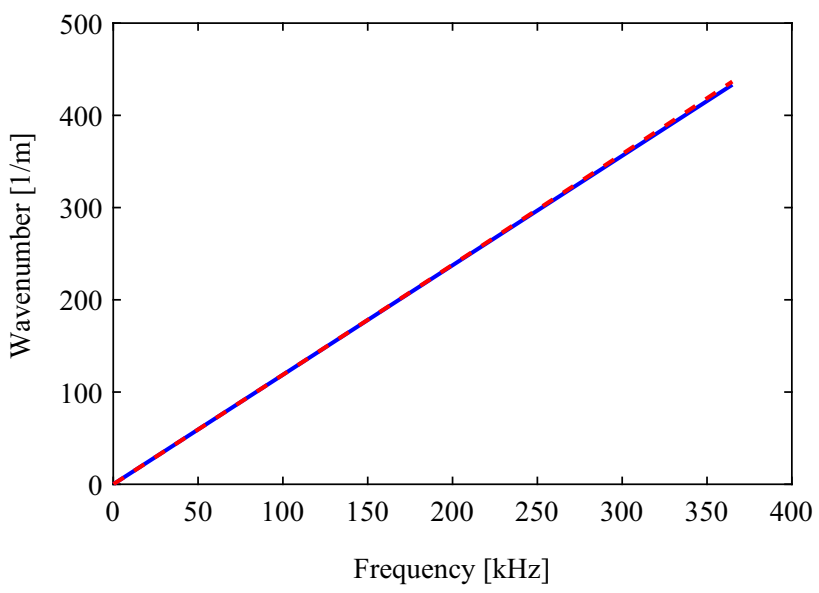

Fig. 6 Dispersion curves for the longitudinal wave in the bar: Analytical(-), WFE(- -)

where $k_{n}$ are longitudinal wavenumbers of the bars determined at each considered frequency $\omega$ as

$k_{n}=\omega \sqrt{\frac{\rho_{n}}{E_{n}}} \quad n \in[1,2, J]$

Equation (34) is solved for the reflection $\mathbf{c}_{\mathbf{1 1}}$ (with $\mathbf{a}_{\mathbf{1}}^{-}=$ $\mathbf{c}_{11} \mathbf{a}_{1}^{+}$) and transmission $\mathbf{c}_{21}$ (with $\mathbf{a}_{2}^{+}=\mathbf{c}_{21} \mathbf{a}_{1}^{+}$) coefficients of the system.

The methodology presented in this work is used to compute the numerical solution of the problem. A segment of each waveguide is modelled using LINK180 FE of length $\Delta=0.001$ in ANSYS. The coupling joint is modelled using three finite elements of similar element size as the waveguides. Then Eq. (12) is solved to obtain the WFE wave dispersion properties. Equation (33) is solved for the WFE/FE wave interaction coefficients. Comparison of the presented WFE/FE predictions and the analytical results are presented in Figs. 6 and 7 respectively. Excellent agreements are observed in the results. Correlation of the transmission coefficient results is good but with little deviation at higher frequencies. This is as a result of FE discretisation whose accuracy limit is recommended to satisfy [37]

$|k \Delta| \leq 1$ 
Fig. 7 Wave interaction coefficients for two collinear bars coupled through a finite bar: analytical(-), WFE-FE(- -). a Reflection coefficient. b Transmission coefficient

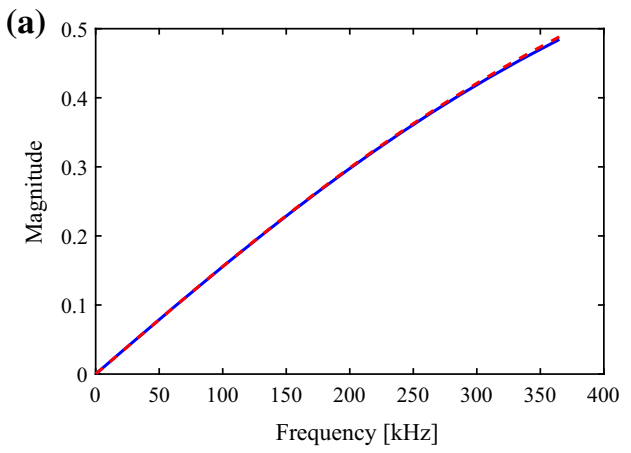

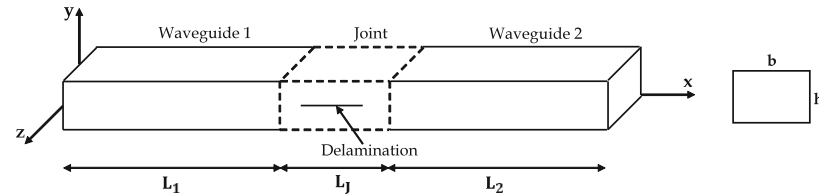

Fig. 8 Schematic illustration of a delaminated beam discretised as a system of two healthy beams connected through a delaminated beam (coupling joint)

As a result of this, for a particular element size $\Delta$, FE discretisation error increases with frequency because wavenumber increases as frequency increases (as shown in Fig. 6). Hence, the deviation observed at higher frequencies between the numerical and analytical results can be attributed to FE discretisation error. This error can be subdued using smaller element size $\Delta$ but at a higher computational cost.

\subsubsection{Delaminated Beam}

The presented methodology is next validated using delaminated continuous aluminium beam having a uniform crosssectional area with $L_{1}=L_{2}=0.2, L_{J}=0.001, b=0.003$ and $h=0.001$. The beam is made of aluminium with material properties $E=70 \times 10^{9}, \rho=2600$ and $v=0.3$. The continuous beam is discretised as a system of two healthy waveguides connected through a delaminated coupling joint as shown in Fig. 8. The beam supports propagating longitudinal, in-plane bending, out-of-plane bending and torsional waves.

A segment of each waveguide is modelled using SOLID185 finite element of length $\Delta=0.001$ in ANSYS. The coupling joint is modelled using similar segment length as the waveguides. The through width delamination in the coupling joint is modelled using the stiffness reduction method (Sect. 3.2) with a reduction factor $\beta=0.01$. Equation (12) is solved to obtain the WFE wave dispersion properties. Then the WFE-FE reflection and transmission efficiencies are calculated as the absolute square of the reflection and transmission coefficients obtained through Eq. (33).

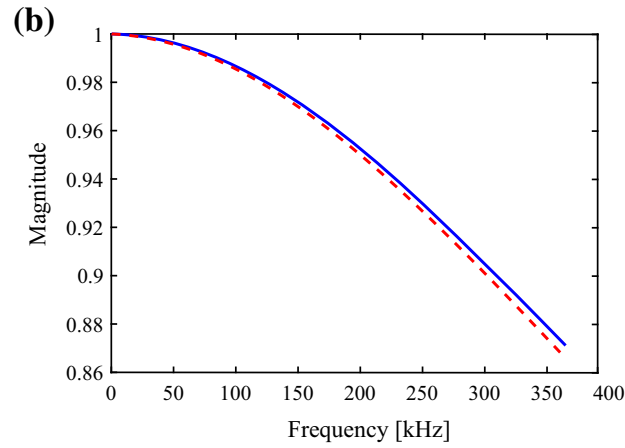

Waves dispersion properties, and reflection and transmission efficiencies of the system are obtained analytically as in $[15,38]$. These are compared to the WFE/FE predicted results as shown in Figs. 9 and 10. The analytical and numerical predictions are in very good agreement.

The coupling joint is undamped, i.e. it is of real-valued elastic and shear moduli. As a conservation of energy condition, the algebraic sum of reflection and transmission efficiencies of a lossless (undamped) structural segment equals unity. As observed in Fig. 10, conservation of energy condition is satisfied for all presented waves as sums of reflection and transmission efficiencies are ones. This further establishes the validity of the presented methodology. Also observed in the waves transmission and reflection results is the fact that the incident waves in waveguide 1 is transmitted or reflected through the coupling joint into waves of the same type without any form of mode conversion. This is expected in waveguides collinearly connected through a joint as waves will be fully transmitted without reflection and modes conversion. Reflection observed is solely as a result of damage in the coupling joint.

\subsubsection{Notched Plate}

Validity of the presented methodology is further proven using notched plate of thickness $2 \mathrm{~d}=0.003$ and length $L=0.6$. The plate is made of mild steel $\left(E=210 \times 10^{9}, \rho=7850\right.$ and $v=0.29$ ) and has uniform area throughout its cross sections.

Based on the presented methodology, the plate can be discretised as a system of two pristine waveguides $\left(L_{1}=\right.$ $\left.L_{2}=0.295\right)$ connected through a notched coupling joint $\left(L_{J}=0.01\right)$ as shown in Fig. 11. Plane strain condition is assumed.

ANSYS is used to model a segment (of length $\Delta=0.001$ ) of each waveguide with PLANE 182 (4-noded quadrilateral finite elements with two translational DoFs per node) FEs. The segment of each waveguide is meshed across its width using 12 elements. Similar element size used for the waveguide segments is repeated for the coupling joint, thereby meshing the joint using 120 elements. 
Fig. 9 Dispersion curves for the beam: Present methodology (-) and Analytical results (o). a Bending wave about $y$-axis. b Bending wave about $\mathrm{z}$-axis. c Torsional wave. d Longitudinal wave

Fig. 10 Reflection and transmission efficiencies of the delaminated beam joint. Present methodology: reflection (o), transmission $(*)$. Analytical results: reflection (- -), transmission (-). Conservation of energy (... ). a Bending wave about y-axis. b Bending wave about $\mathrm{z}$-axis. $\mathbf{c}$ Torsional wave. d Longitudinal wave
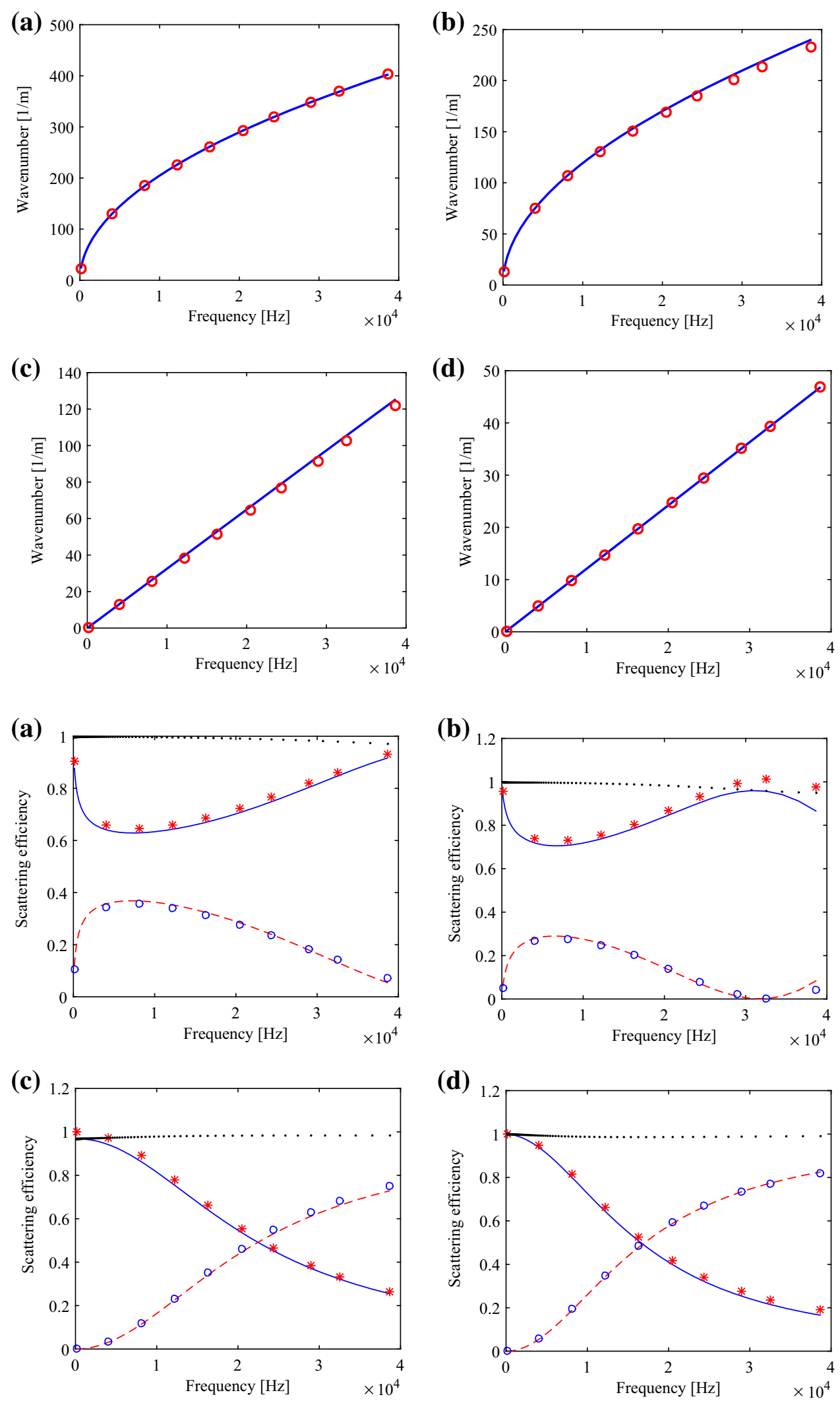


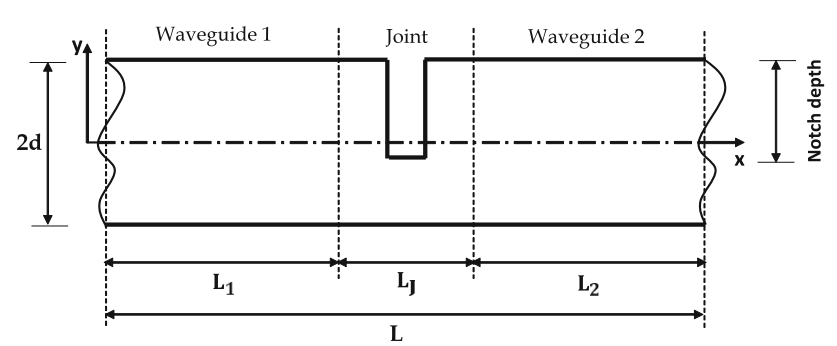

Fig. 11 Schematic illustration of a notched plate discretised as a system of two healthy waveguides connected through a notched coupling joint

Waves reflections are obtained from notches, of depths $0.0005,0.001$ and 0.002 , which are respectively 17,33 and $67 \%$ of the plate thickness. Uniform notch (of width 0.0005 ) is used in all cases. The notches are modelled using the element deletion approach presented in 3.1.

In practice, the wave reflection calculation can be made by a full FE transient simulation. The WFE computed eigenvectors can be windowed and then applied as time-dependent harmonic displacement boundary conditions (of excitation frequency $\omega$ ) at one of the extreme cross-sections of the plate. In this case, the entire plate is modelled as one plate instead of a system of waveguides and coupling joint as in the case of the presented WFE-FE approach.

Results obtained through the WFE-FE methodology are compared with that of the full FE transient simulation and the experimental measurements presented in [39]. Modelling parameters used for the WFE/FE methodology are chosen to match those used for the full FE simulation and the experimental measurements in [39].

Good agreement is observed among the WFE-FE, full FE and experimental results as shown in Fig. 12. It is also worthy to state that the developed methodology is more efficient (than the full FE approach) for predicting wave scattering (reflection and transmission) from damage within structural waveguides for the following noted reasons. First, model size and computational time. Finite element mesh of the plate consists of 7200 elements and 15,626 DoFs in the full FE model against a total of 144 elements (12 for each waveguide and 120 for the joint) and $390 \mathrm{DoFs}$ in the presented WFE-FE model. Solving the full FE model requires a computational time of about 105 min compared to the WFE-FE model which is solved under $5 \mathrm{~min}$. Therefore, a great deal of computational time and hence cost is saved by the WFE-FE approach. Another noted point is in terms of the complexity of the structural system. Full FE model mostly assume plane strain condition in order to simplify and reduce model size of a structural system. In this manner, some propagating waves especially those along the suppressed axis might not be captured. However, the presented WFE/FE approach can be applied for analysing wave interaction in complex structural systems (such as composite structures and structural networks) with low computational size and cost.
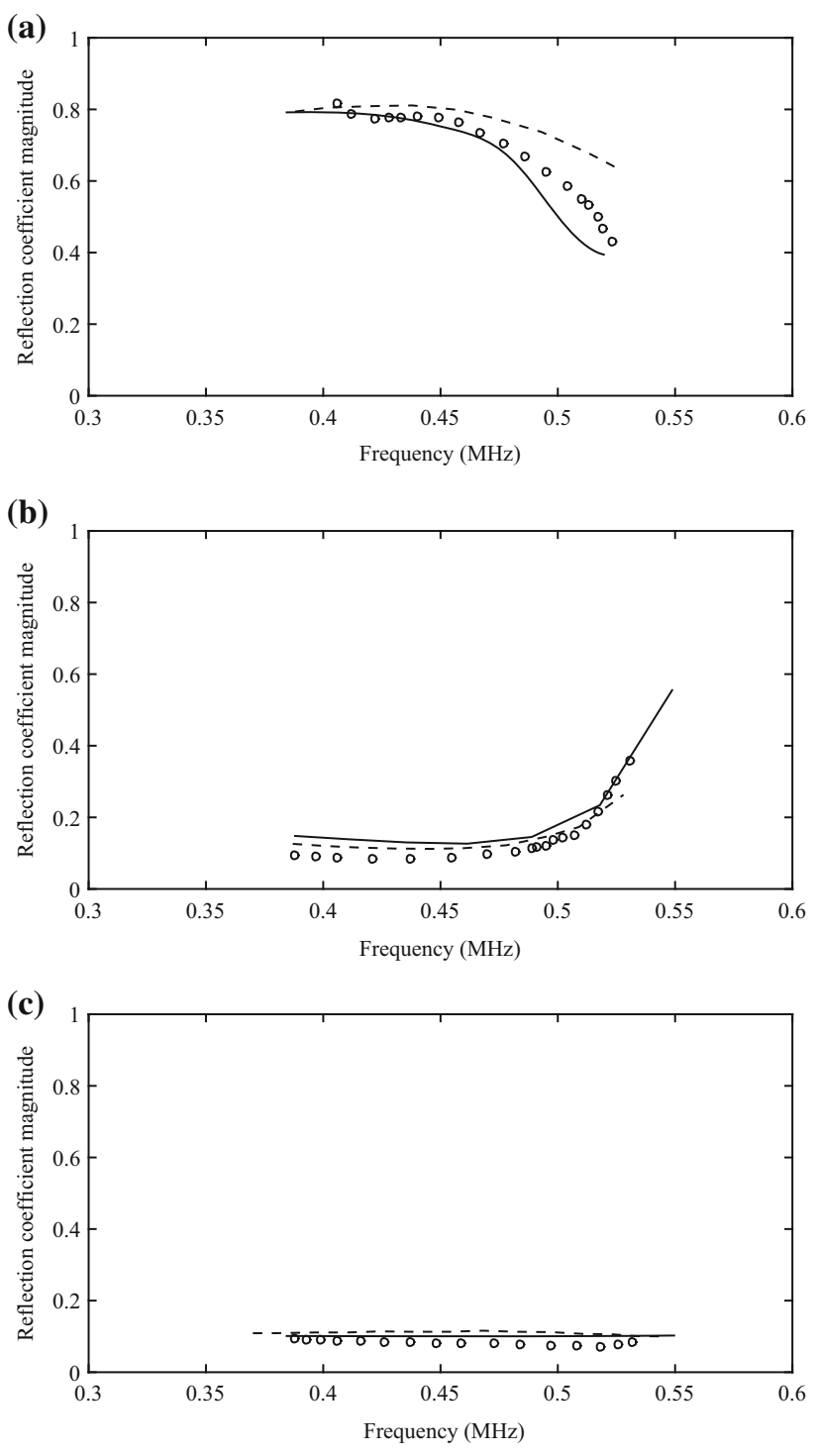

Fig. 12 Comparison of the wave reflection coefficients from notch of varying depths: Presented WFE-FE methodology (-), Full FE predictions (- -) and Experimental measurements (o) in [39]. a $67 \%$ notch depth. b $33 \%$ notch depth. c $17 \%$ notch depth

\subsection{Test Case Studies}

\subsubsection{Transversely-Isotropic Beam}

In this example, a cracked transversely-isotropic laminate having a uniform cross-sectional area $(b=0.01$ and $h=$ $0.005)$ is considered. The beam is defined as a system of two pristine beams $\left(L_{1}=L_{2}=0.2\right)$ connected through a cracked beam $\left(L_{J}=0.004\right)$ as shown in Fig. 13. Each beam comprises five layers of glass-epoxy whose material properties are $E_{x}=E_{y}=68 \times 10^{9}, E_{z}=40 \times 10^{9}$, $G_{x y}=3.6 \times 10^{9}, G_{y z}=G_{x z}=1.2 \times 10^{9}, v_{x y}=0.25$, $v_{y z}=v_{x z}=0.334$ and $\rho=2700$. 
Fig. 13 Schematic illustration of the WFE-FE configuration of a pressurised transversely-isotropic laminated beam

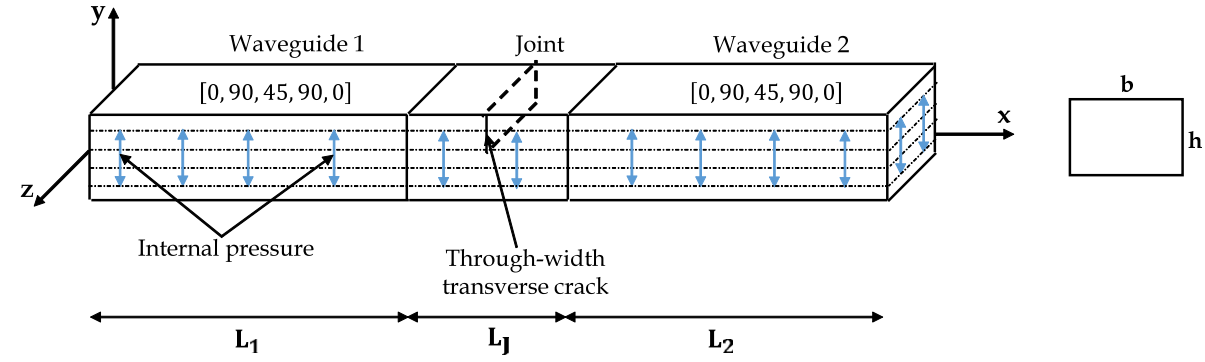

Fig. 14 Dispersion curves for the transversely-isotropic beam: non-pressurised (-), internal pressure $p=0.1 \mathrm{GPa}(-+), 0.5$ $\mathrm{GPa}(-\mathrm{x}), 1.0 \mathrm{GPa}(-*)$ and 1.5 $\mathrm{GPa}(-0)$. a Bending wave about $\mathrm{y}$-axis. b Bending wave about Z-axis. c Longitudinal wave. d Torsional wave
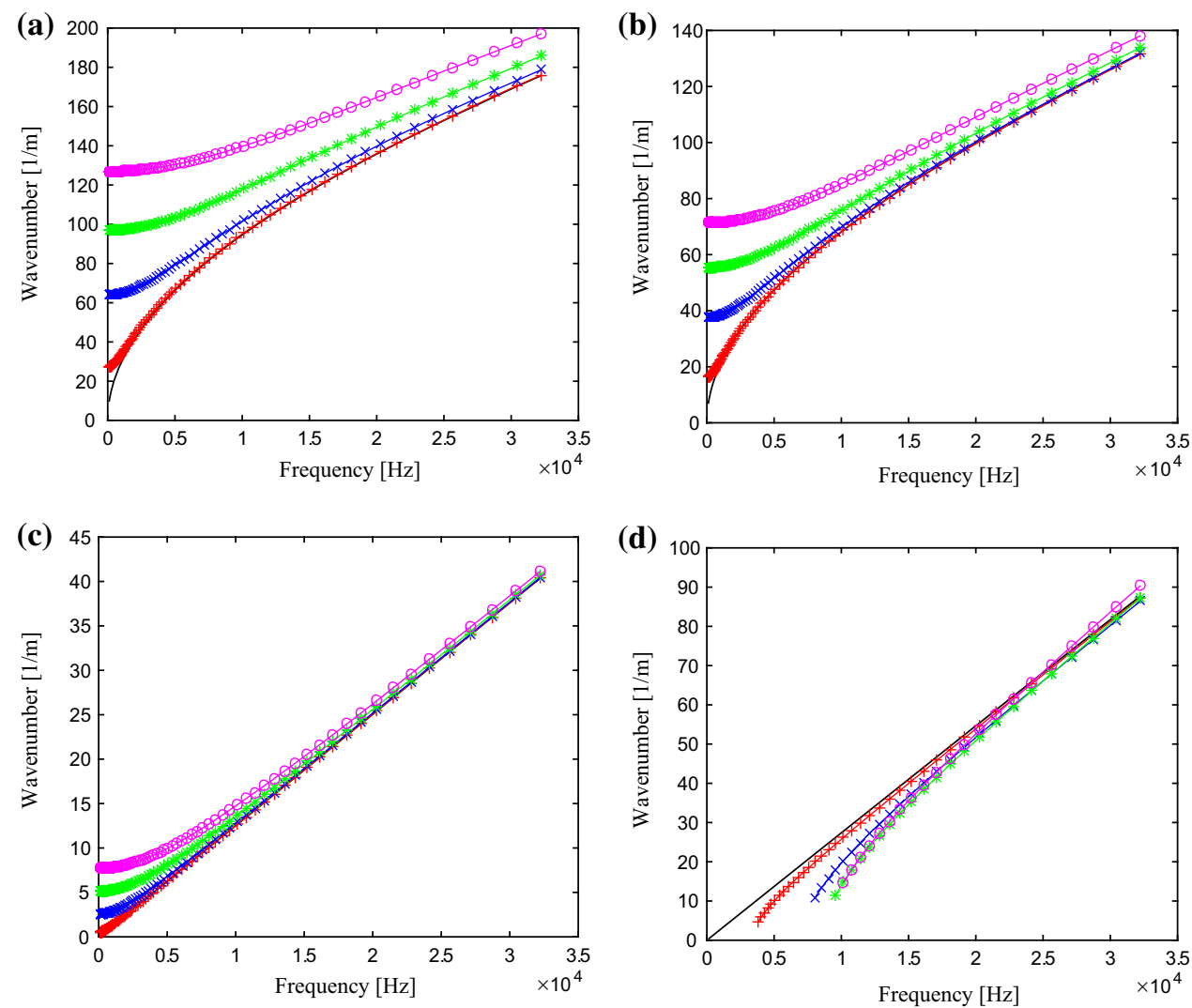

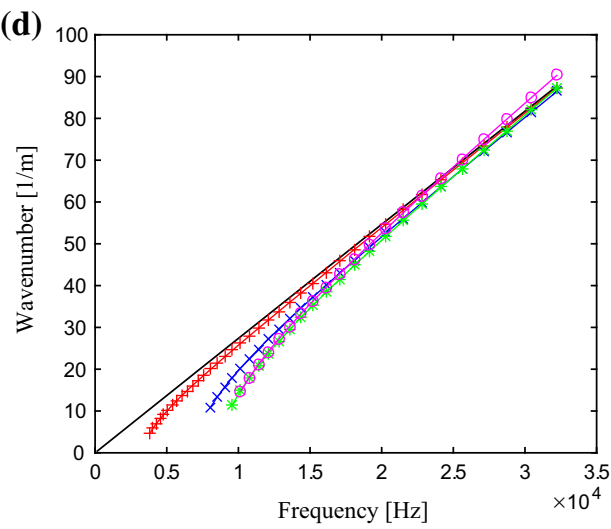

A segment (of length $\Delta=0.001$ ) of each waveguide is modelled in ANSYS with 40 SOLID185 finite elements using cubed sized elements of length 0.001 . Using similar element size, the coupling joint is modelled with 160 finite elements. The crack within the joint is modelled using the node duplication approach (Sect. 3.3). Two crack scenarios, of depths 0.001 and 0.002 , are considered. These are respectively equivalent to 20 and $40 \%$ of the total depth of the beam. The cracks are through-width and located at mid length of the joint.

Each beam is pre-stressed using uniform internal pressure. The pressure is applied across the surfaces of the three internal layers of the laminated beam as shown in Fig. 13. Five different pressure scenarios are considered; non-pressurised case and pressurised cases with applied pressure $p=0.1$ $\mathrm{GPa}, 0.5 \mathrm{GPa}, 1.0 \mathrm{GPa}$ and $1.5 \mathrm{GPa}$.
Dispersion curves for each waveguide are obtained by solving Eq. (12) within frequency range $\omega=[1.0 \times$ $\left.10^{2}-3.3 \times 10^{4}\right] \mathrm{Hz}$. The dispersion curves are presented in Fig. 14. Four propagating modes at each frequency are obtained for the non-pressurised waveguide. For the pressurised waveguide, there are three propagating modes (y-axis bending wave, $\mathrm{z}$-axis bending wave and longitudinal wave) at low frequency range. Fourth mode (torsional wave) cuts on at $\omega=3.8 \times 10^{3}, 8.1 \times 10^{3}, 9.6 \times 10^{3}$ and $1.0 \times 10^{4}$ $\mathrm{Hz}$ in the $0.1,0.5,1.0$ and $1.5 \mathrm{GPa}$ pressurised waveguides. In the low frequency range, the wavenumbers of the pressurised waveguide are significantly different compared to the non-pressurised one. An average difference of about $32 \%$ per $0.1 \mathrm{GPa}$ is observed for the bending waves at low frequency range. Differences of about 20 and $11 \%$ are observed for the longitudinal and torsional wavenumbers. Increase in the wavenumbers can be attributed to reduc- 
Fig. 15 Wave reflection coefficients for the transversely-isotropic laminate with $20 \%$ depth crack: non-pressurised (-), internal pressure $p=0.1 \mathrm{GPa}(-+), 0.5$ $\mathrm{GPa}(-\mathrm{x}), 1.0 \mathrm{GPa}(-*)$ and 1.5 GPa (-o). a Bending wave about y-axis. b Bending wave about $\mathrm{z}$-axis. c Longitudinal wave. $\mathbf{d}$ Torsional wave
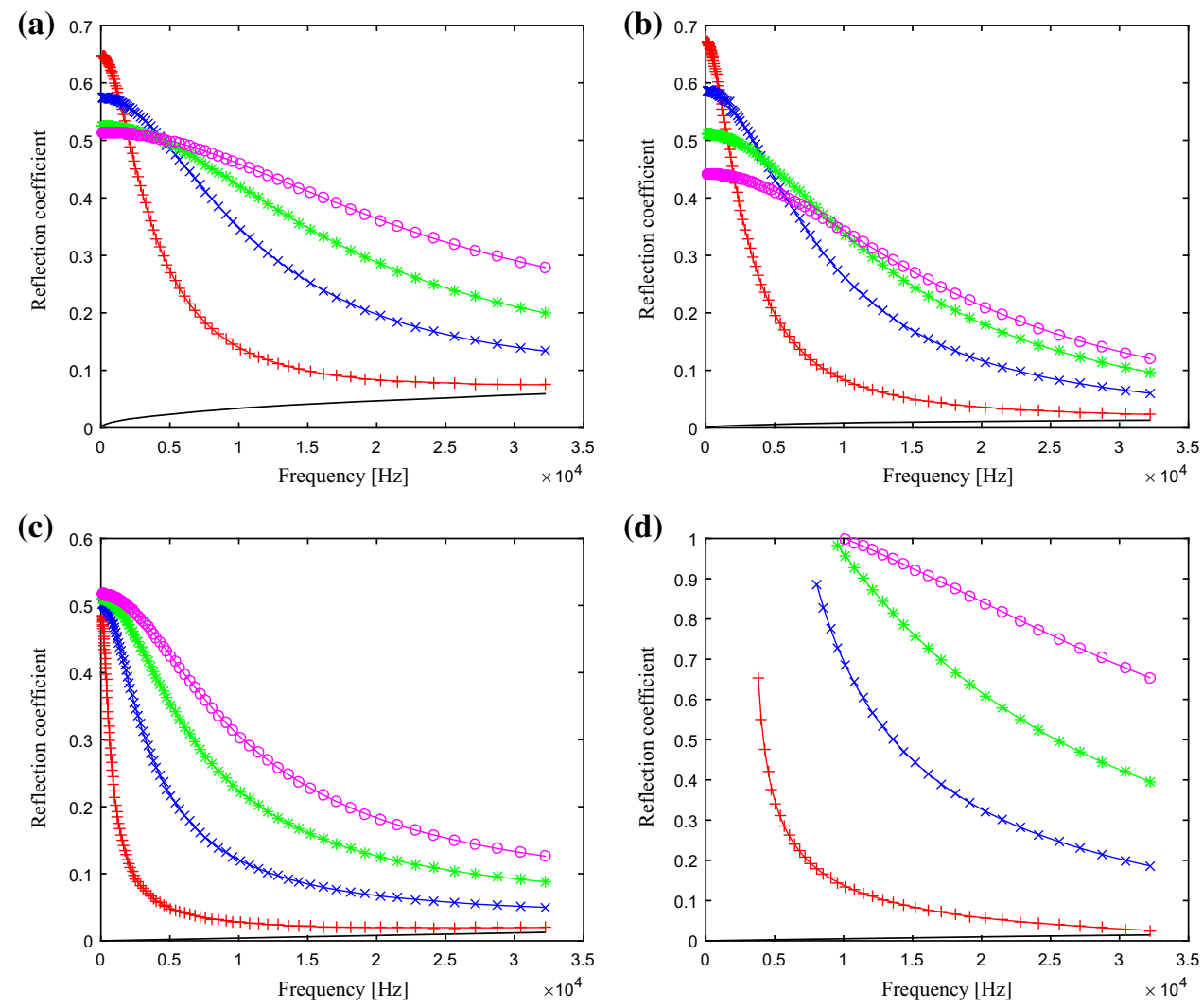

tion in loss factor of the waveguide due to an increase in strain energy as a result of the applied pressure. The difference in wavenumbers (of the non-pressurised and pressurised waveguides) diminishes gradually as frequency gets higher.

Equation (33) is solved for the wave interaction coefficients from the cracked coupling joint. Reflection coefficients for the 20 and the $40 \%$ depth cracks are presented in Figs. 15 and 16. As observed from the results, magnitude of the wave reflection coefficient increases with depth of crack.

With respect to applied pressure, it can be seen that wave reflection coefficient magnitudes increase with an increase in the magnitude of applied pressure. Average increments of about $45,20,25$ and $90 \%$ per $0.1 \mathrm{GPa}$ are respectively observed for the $\mathrm{y}$-axis bending wave, the $\mathrm{z}$-axis bending wave, the longitudinal and the torsional wave.

Consequently from the presented results, magnitudes of wave constants and interaction coefficients are generally boosted by applied pressure. This can therefore be used to detect micro structural defects which may not be easily detected under non-pressurisation scenario. Application of pre-stressing (through pressurisation) as a damage detection method is further examined using a sandwich laminate with micro delamination as presented in next section.

\subsubsection{Sandwich Beam}

In the final test case, a delaminated sandwich beam is considered. The asymmetric sandwich beam consists of carbon epoxy facesheets $\left(\rho=3500, E_{x}=E_{y}=E_{z}=54 \times 10^{9}\right.$, $G_{x y}=2.8 \times 10^{9}, G_{y z}=G_{x z}=1.0 \times 10^{9}, v_{x y}=v_{y z}=$ $\left.v_{x z}=0.3, h_{s 1}=0.002, h_{s 2}=0.001\right)$ and an isotropic core $\left(E=70 \times 10^{9}, \rho=50, v=0.3, h_{c}=0.01\right)$. The beam's cross-section $(b=0.005, h=0.013$ is constant throughout and are fixed at both ends.

The delaminated beam is discretised as two healthy beams $\left(L_{1}=L_{2}=0.2\right)$ coupled through a delaminated joint $\left(L_{J}=\right.$ 0.004) as shown in Fig. (17). The beams are modelled in ANSYS using SOLID185 elements. Cubed sized elements of length 0.001 are used to model the facesheets, while elements of size $0.001 \times 0.002 \times 0.001$ are employed for modelling the core. As a result, 40 finite elements are used for the WFE model of a periodic segment (of length $\Delta=0.001$ ) of each waveguide and 160 elements for the full FE model of the coupling joint.

Interlaminar delamination, along the interface of the upper facesheet and the core, is considered. Two delamination scenarios (20 and $40 \%$ of the beam width) are examined. They are both of length 0.002 (symmetrically located about the mid length of the joint).

As in the previous example, each beam is pre-stressed using uniform internal pressure. The pressure is applied 
Fig. 16 Wave reflection coefficients for the transversely-isotropic laminate with $40 \%$ depth crack: non-pressurised (-), internal pressure $p=0.1 \mathrm{GPa}(-+), 0.5$ $\mathrm{GPa}(-\mathrm{x}), 1.0 \mathrm{GPa}(-*)$ and 1.5 $\mathrm{GPa}(-\mathrm{o})$. a Bending wave about y-axis. b Bending wave about $\mathrm{z}$-axis. c Longitudinal wave. d Torsional wave

Fig. 17 Schematic representation of the WFE-FE configuration of a pressurised sandwich beam (a)

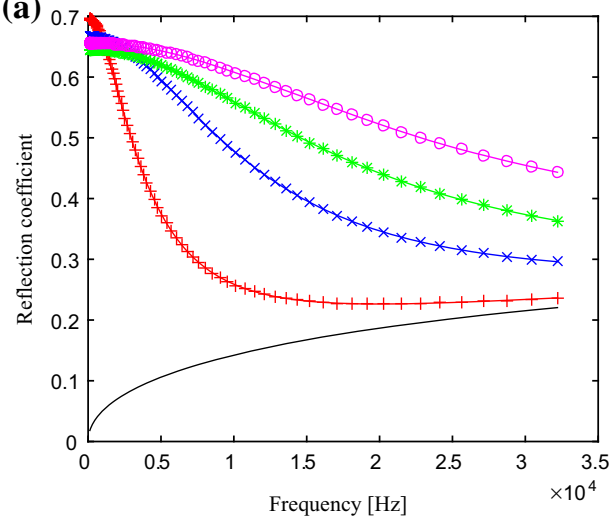

(c)

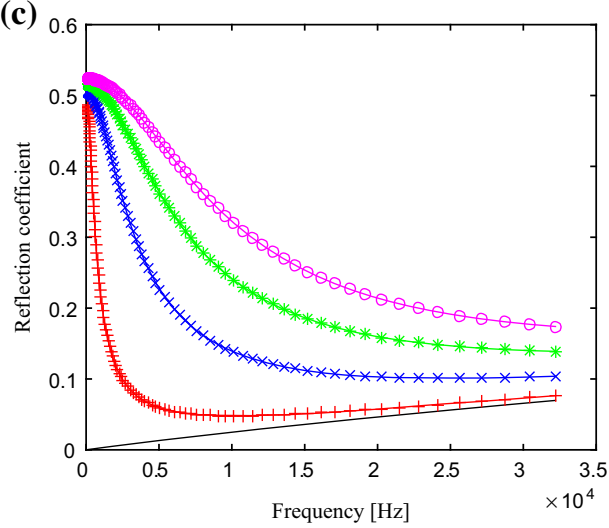

(b)

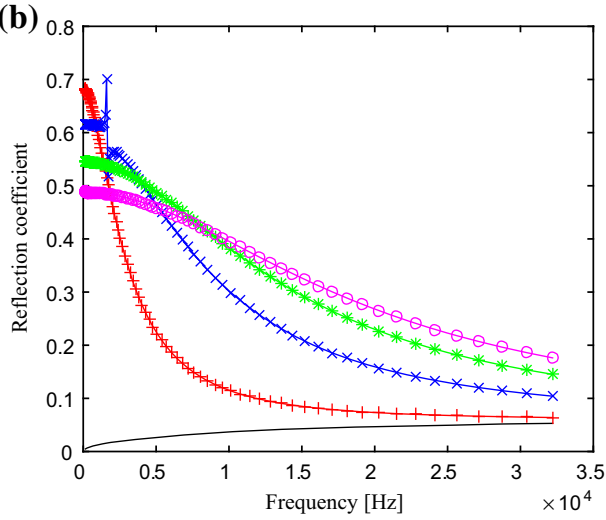

(d)

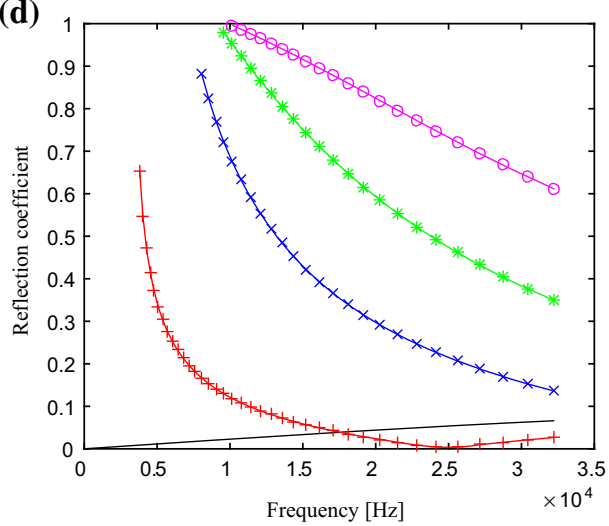

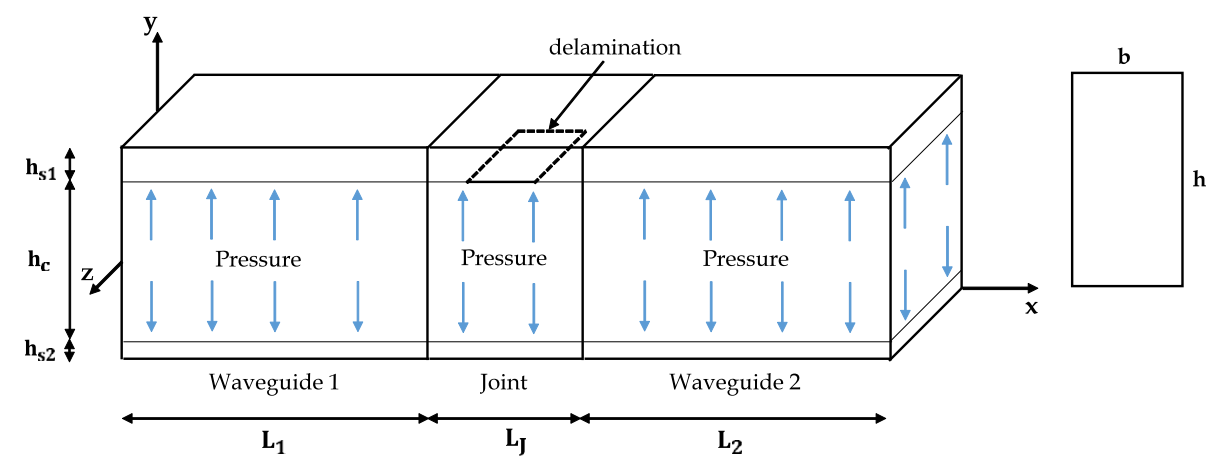

across the surfaces of the sandwich core as shown in Fig. 17. Six different pressure scenarios are examined; one with no pressure applied and the rest with applied pressure of 0.1 , $0.2,0.3,0.4$ and $0.5 \mathrm{GPa}$ per $(0.001 \times 0.002)$ cross sectional area.

Equation (12) is solved for the dispersion curves of the waveguide. Four propagating waves are obtained, with the remaining waves being nearfield waves. Presented in Fig. 18 are the dispersion curves of the propagating waves as a function of frequency. The curves are presented for each of the seven pressure scenarios. For the pressurised waveguide, a smooth, nearly linear behaviour (with regards to the wavenumbers) is observed as a function of frequency. This is observed up until a certain frequency, then a rapid rise takes place. This behaviour is similar for all the pressure scenarios. However, the frequency at which the rapid rise is observed varies with the applied pressure. It is observed at about $1.0,1.6,2.2,2.8$ and $3.4 \mathrm{kHz}$ respectively in the 0.1 , $0.2,0.3,0.4$ and $0.5 \mathrm{GPa}$ pressurised waveguides. There is also a steady increase in the wavenumbers as a function of applied pressure. An average increase of about $27 \%$ per 0.1 $\mathrm{GPa}$ is observed at each frequency in the low frequency range. It should be noted that the differences are normalised as frequency gets higher and tend to become equal irrespective of the magnitude of pressure applied.

Equation (33) is solved for the wave reflections coefficients from the delamination. Figure 19 presents the coefficient magnitudes of wave reflections from the 20 and the 
Fig. 18 Dispersion curves for the sandwich beam:

non-pressurised (-), pressure $p=0.01 \mathrm{GPa}(--), 0.1 \mathrm{GPa}$ (-+), $0.2 \mathrm{GPa}(-\mathrm{x}), 0.3 \mathrm{GPa}\left(-^{*}\right)$, $0.4 \mathrm{GPa}(-\mathrm{o})$ and $0.5 \mathrm{GPa}(->)$. a Bending wave about $\mathrm{y}$-axis. $\mathbf{b}$ Bending wave about $\mathrm{z}$-axis. $\mathbf{c}$ Longitudinal wave. d Torsional wave
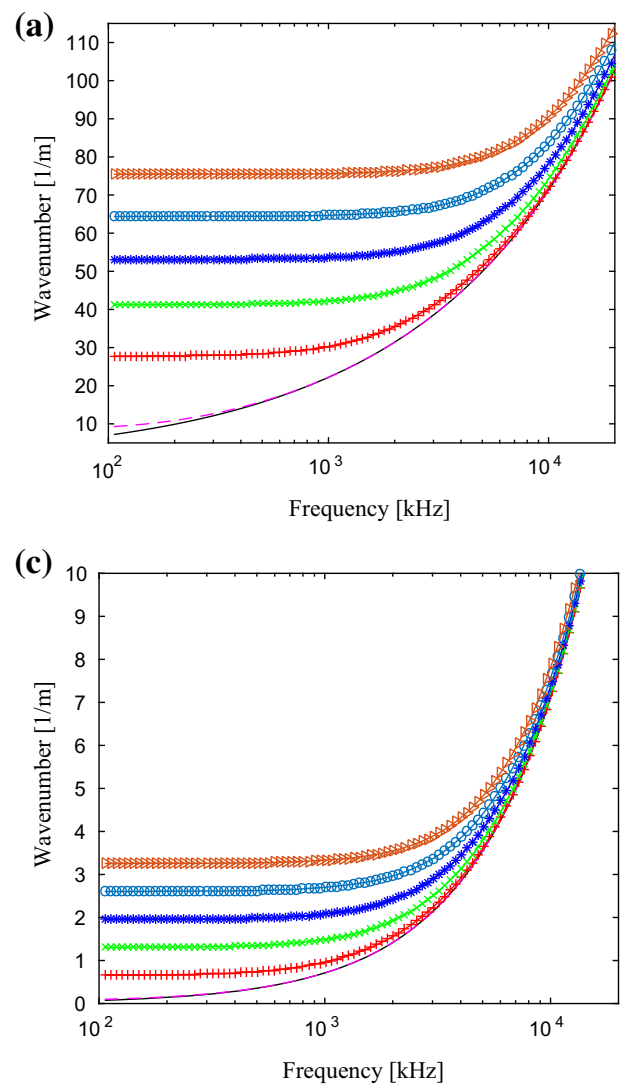

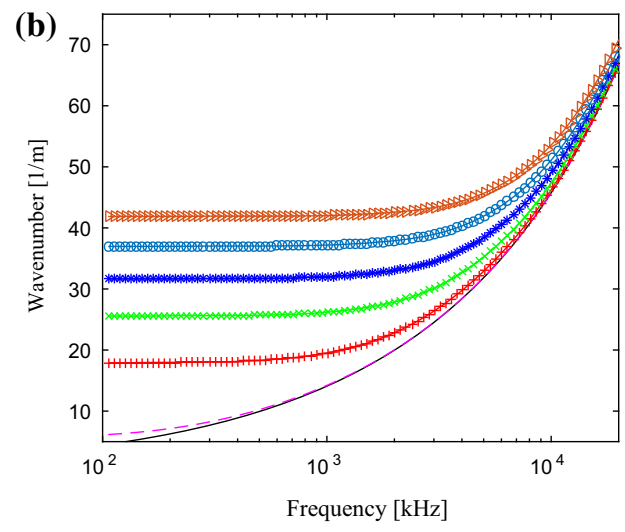

(d)

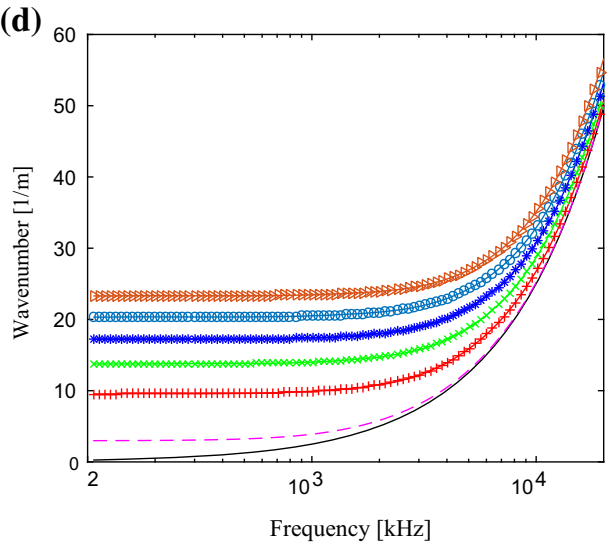

$40 \%$ widths delaminations in the non-pressurised sandwich beam. Due to the minute severity of the considered delaminations, little reflection magnitudes are obtained within the considered frequency range. It can also be seen that negligible differences are observed between both delamination scenarios for all propagating wave types.

The reflection coefficients magnitude becomes greatly significant in the pressurised system as shown in Fig. 20. Compared to the non-pressurised system, there is an average change of about $50-70 \%$ in the low frequency range and of about $10-25 \%$ in the high frequency range. This can be explained by the fact that structural pre-stressing brings about change in the loss factor of the structure. In general, this consequently affects the magnitude of wave propagation properties.

As a function of frequency, the reflection magnitudes are constant over the low frequency range, then reduce slowly over the mid frequency range before reducing rapidly over the high frequency range. This behaviour is similar for all wave types and at each applied pressure.

Variation of the reflection coefficient magnitudes as a function of applied pressure is extensively examined at different frequency within each of the identified frequency ranges i.e., at which coefficient magnitude is constant (e.g., $0.2 \mathrm{kHz}$ ), slightly reduces (e.g., $0.8 \mathrm{kHz}$ ) and rapidly reduces (e.g., 6.4 $\mathrm{kHz}$ ) with respect to frequency.
At $0.2 \mathrm{kHz}$ (Fig. 21), there is a proportional reduction of about $28 \%$ per $0.1 \mathrm{GPa}$ in the reflection coefficient of the $\mathrm{y}$-axis bending wave. Similar trend is observed for the $\mathrm{z}$ axis bending wave. An average increment of about $30 \%$ per $0.1 \mathrm{GPa}$ is observed for the torsional wave while a slight steady increment of about $2 \%$ per $0.1 \mathrm{GPa}$ is observed for the longitudinal wave.

At $0.8 \mathrm{kHz}$ (Fig. 22), the reflection magnitudes of the $\mathrm{x}$ and $y$ axes bending waves increase with the applied pressure up to $1 \mathrm{GPa}$. Beyond this pressure, reduction in the magnitudes is observed. Longitudinal and torsional waves both show similar trends. There is an increase in their coefficient magnitudes with respect to applied pressure. Increment obtained for the torsional wave is however more than that of the longitudinal wave.

Unlike at 0.2 and $0.8 \mathrm{kHz}$, the reflection coefficient magnitudes of the bending waves at $6.4 \mathrm{kHz}$ (Fig. 23) increase with respect to applied pressure. The longitudinal and the torsional waves maintain similar trend as in 0.2 and $0.8 \mathrm{kHz}$.

Similar to the non-pressurised system, it is noted that there is no significant difference observed in the reflection coefficients (with respect to frequency and applied pressure) of the $20 \%$ width delaminated joint compared to that of the $40 \%$ width delamination. 

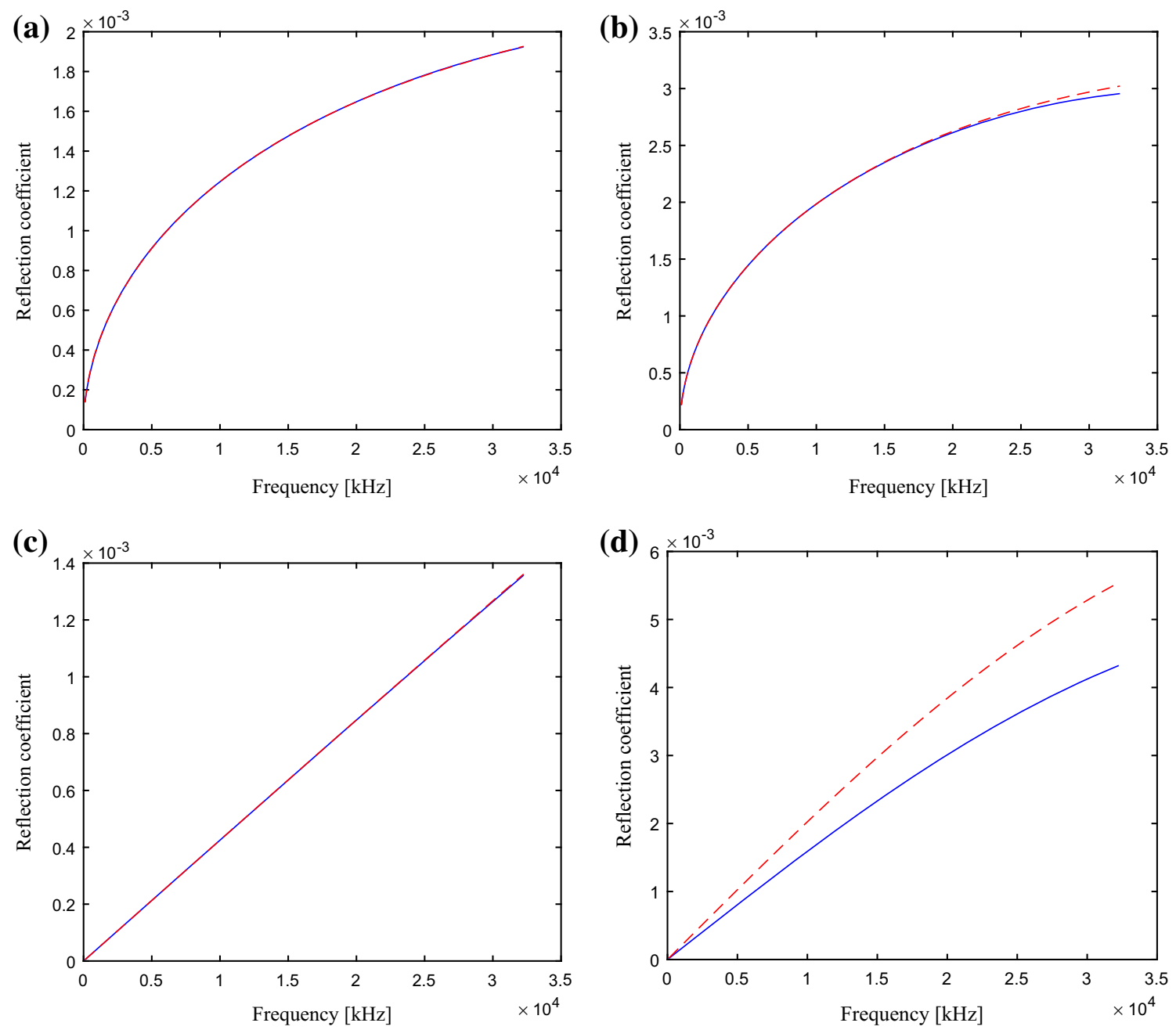

Fig. 19 Wave reflection coefficient magnitude from the $20 \%$ (-) and the $40 \%$ (- -) widths delaminations in the non-pressurised sandwich beam. a Bending wave about y-axis. b Bending wave about z-axis. c Longitudinal wave. d Torsional wave

\section{Concluding Remarks}

This paper presents a FE-based methodology for quantifying wave interaction with localised structural defects. The scheme can be applied to structures of arbitrary complexity, layering and material characteristics as FE discretisation is employed. The scheme discretises defective structural waveguide into a system of two pristine waveguides connected through a defective coupling joint. The wave propagation properties within the pristine waveguides are coupled to the localised defect in the joint in order to compute the wave reflection and transmission properties for each propagating wave mode in the system. The presented methodology also examine the effect of pre-stress on the wave propagation and transmission properties of pressurised structures. The principal outcome of the work can be summarised as follows: (a) The presented approach is validated with analytical and full FE transient response predictions. Very good agreement is observed.

(b) The approach is able to predict the dispersion properties of an arbitrarily complex structure as well as the reflection and transmission coefficients of the wave interaction with defects within the structure.

(c) The approach also successfully examined the effect of pre-stress on the wave properties of pressurised structures. It was shown that pressurisation can be used to detect micro defects which may be too small to detect under no pressurisation.

Future development focuses on implementing multi-scale damage models in order to capture the nonlinear mechanics of advanced damage. 
Fig. 20 Wave reflection coefficient magnitude from the $20 \%$ (-) width delamination within the sandwich beam: non-pressurised (-), pressure $p=0.01 \mathrm{GPa}(--), 0.1 \mathrm{GPa}$ $(-+), 0.2 \mathrm{GPa}(-\mathrm{x}), 0.3 \mathrm{GPa}(-*)$, $0.4 \mathrm{GPa}(-\mathrm{o})$ and $0.5 \mathrm{GPa}(->)$. a Bending wave about $y$-axis. b Bending wave about $\mathrm{z}$-axis. c Longitudinal wave. $\mathbf{d}$ Torsional wave
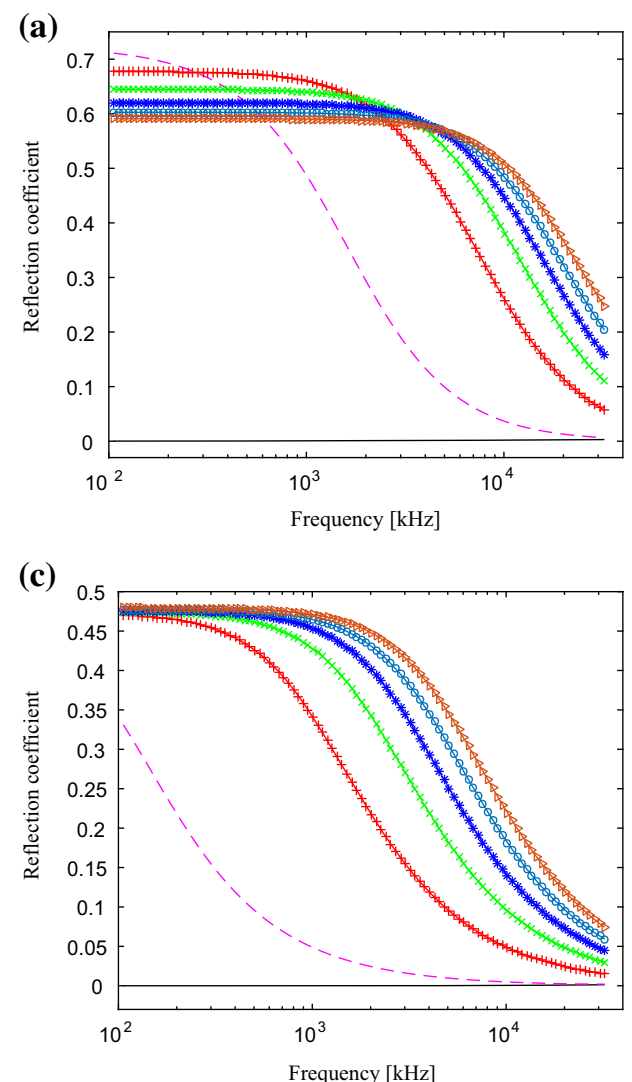

Fig. 21 Wave reflection coefficient magnitude at $0.2 \mathrm{kHz}$ for the pressurised sandwich beam as a function of applied pressure: $20 \%$ (-) width delamination, $40 \%$ (- -) width delamination. a Bending wave about y-axis. b Bending wave about z-axis. c Longitudinal wave. d Torsional wave
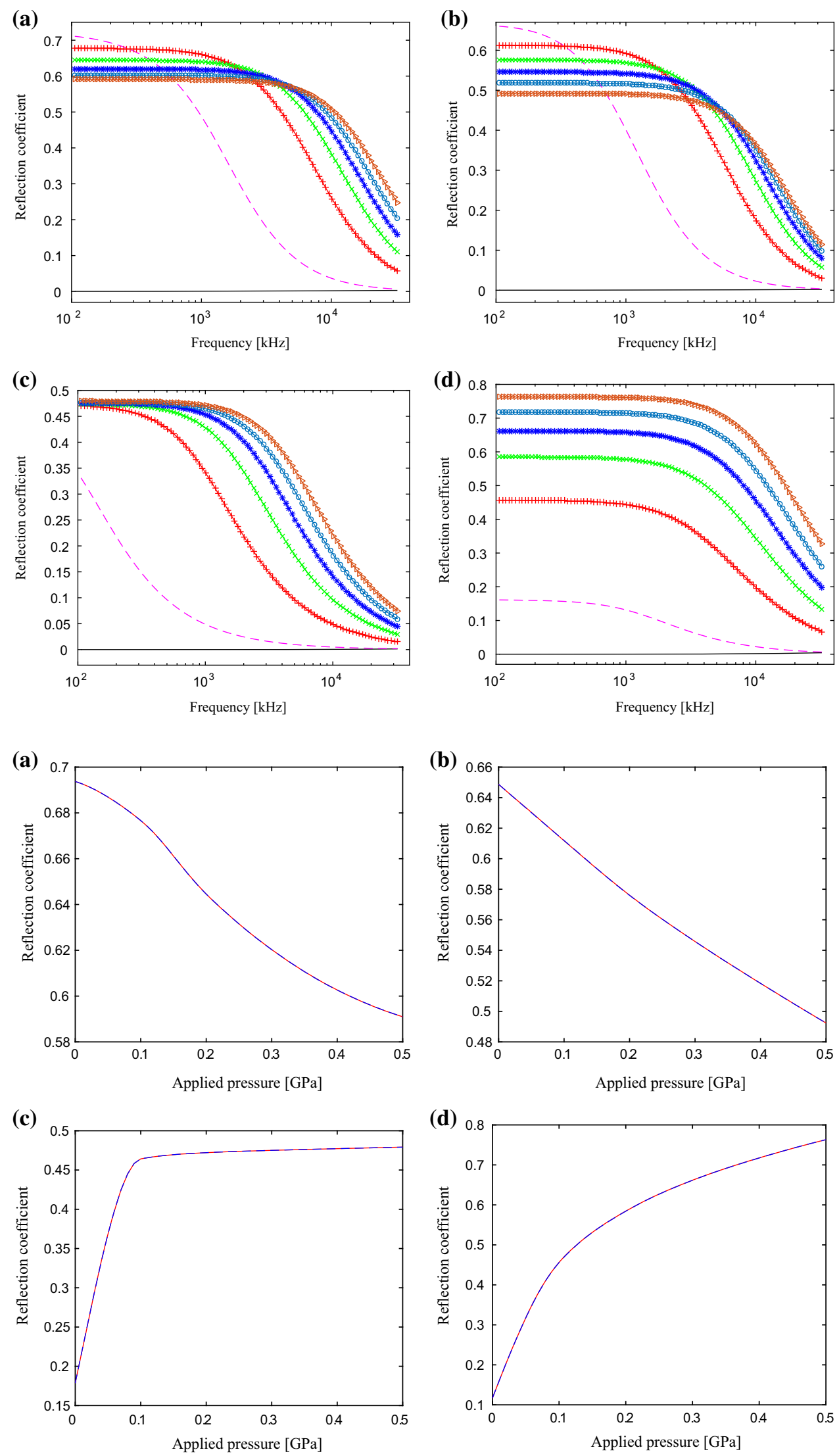
Fig. 22 Wave reflection coefficient magnitude at $0.8 \mathrm{kHz}$ for the pressurised sandwich beam as a function of applied pressure: $20 \%(-)$ width delamination, $40 \%$ (- -) width delamination. a Bending wave about y-axis. b Bending wave about z-axis. c Longitudinal wave. d Torsional wave
Fig. 23 Wave reflection coefficient magnitude at $6.4 \mathrm{kHz}$ for the pressurised sandwich beam as a function of applied pressure: $20 \%$ (-) width delamination, $40 \%$ (- -) width delamination. a Bending wave about $y$-axis. b Bending wave about z-axis. c Longitudinal wave. $\mathbf{d}$ Torsional wave
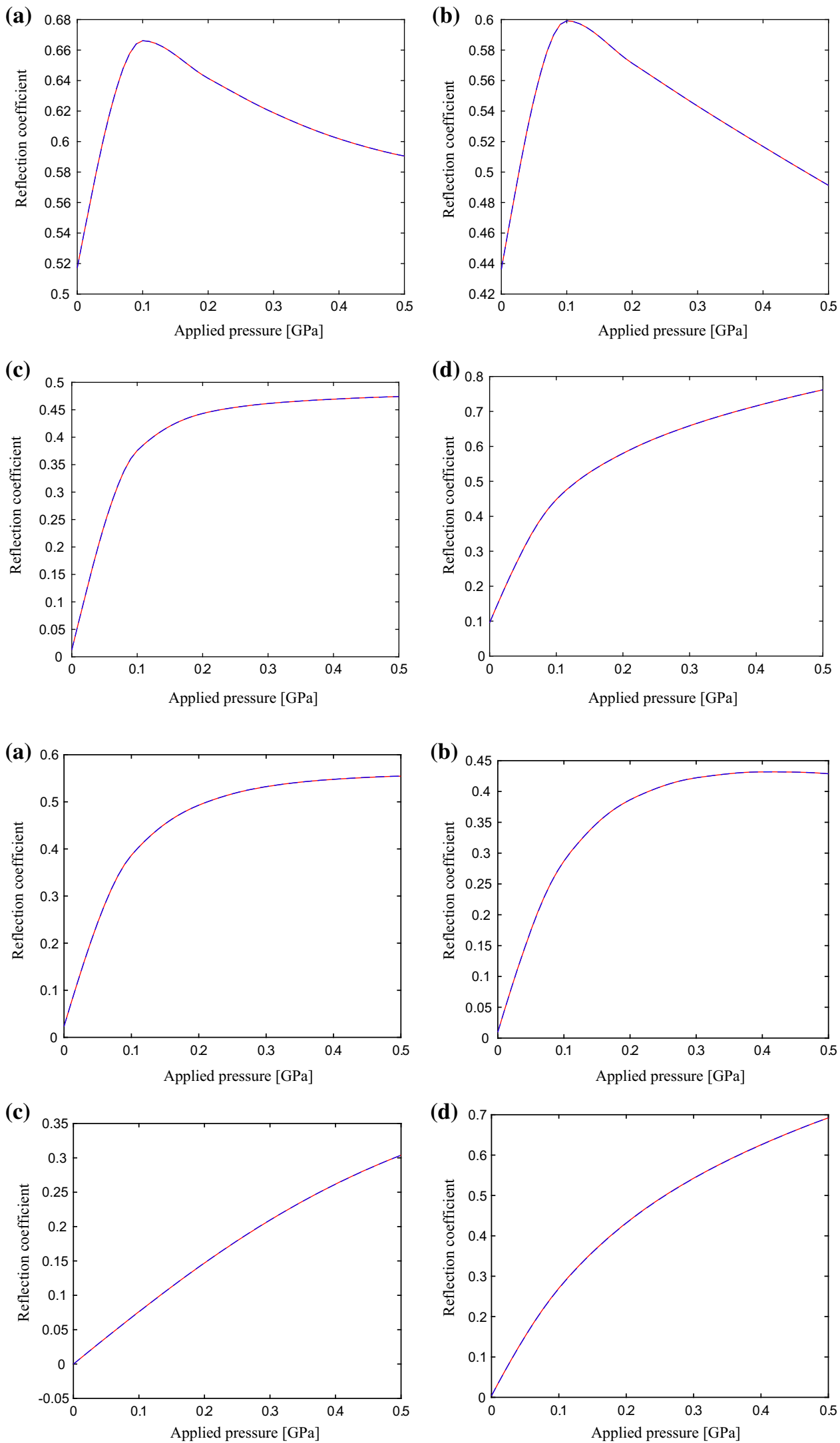
Open Access This article is distributed under the terms of the Creative Commons Attribution 4.0 International License (http://creativecomm ons.org/licenses/by/4.0/), which permits unrestricted use, distribution, and reproduction in any medium, provided you give appropriate credit to the original author(s) and the source, provide a link to the Creative Commons license, and indicate if changes were made.

\section{References}

1. Heslehurst, R.: Defects and Damage in Composite Materials and Structures. CRC Press, Boca Raton (2014)

2. Kessler, S., Spearing, S., Soutis, C.: Damage detection in composite materials using lamb wave methods. Smart Mater. Struct. 11, 269278 (2002)

3. Raju, P.: Acousto-ultrasonic technique for nondestructive evaluation of composites and structures. Acoust. Soc. Am. J. 102, 3082 (1997)

4. Chien, H., Sheen, S., Raptis, A.: An acousto-ultrasonic nde technique for monitoring material anisotropy. In: Thompson, D.O., Chimenti, D.E. (eds.) Review of Progress in Quantitative Nondestructive Evaluation, pp. 1225-1232. Springer, New York (1993)

5. Worlton, D.: Ultrasonic testing with lamb waves. Non-Destr. Test. 15, 218-222 (1957)

6. Rose, J.: Ultrasonic Waves in Solid Media. Cambridge University Press, Cambridge (2004)

7. Donskoy, D., Sutin, A.: Vibro-acoustic modulation nondestructive evaluation technique. J. Intell. Mater. Syst. Struct. 9, 765-771 (1998)

8. Su, Z., Zhou, C., Hong, M., Cheng, L., Wang, Q., Qing, X.: Acousto-ultrasonic based fatigue damage characterization: linear versus nonlinear signal features. Mech. Syst. Signal Process. 45, 225-239 (2014)

9. Lowe, M.: Matrix techniques for modelling ultrasonic waves in multi-layered media. IEEE Trans. Ultrason. Ferroelectr. Freq. Cont. 42, 525-542 (1995)

10. Young, Y., Lin, Y.: Dynamic response analysis of truss-type structural networks: a wave propagation approach. J. Sound Vib. 155, 375-381 (1992)

11. Hutchinson, R., Fleck, N.: The structural performance of the periodic truss. J. Mech. Phys. Solids 54, 756-782 (2006)

12. Mace, B.: Wave reflection and transmission in beams. J. Sounds Vib. 72, 237-246 (1984)

13. Chouvion, B., Popov, A., McWilliam, S., Fox, C.: Vibration modelling of complex waveguide structures. Comput. Struct. 89, 1253-1263 (2011)

14. Yuana, W., Zhoua, L., Yuanb, F.: Wave reflection and transmission in composite beams containing semi-infinite delamination. J. Sounds Vib. 313, 676-695 (2007)

15. Doyle, J.: Wave Propagation in Structures: Spectral Analysis Using Fast Discrete Fourier Transforms. Springer, New York (1997)

16. Hosten, B., Castaings, M.: Finite elements methods for modeling the guided waves propagation in structures with weak interfaces. J. Acoust. Soc. Am. 117, 1108-1113 (2005)

17. Pialucha, T., Cawley, P.: The reflection of ultrasound from interface layers in adhesive joints. Review of Progress in Quantitative Nondestructive Evaluation, pp. 1303-1309. Springer, Boston (1991)

18. Leung, R., Pinnington, R.: Wave propagation through right-angled joints with compliance: longitudinal incidence wave. J. Sound Vib. 153, 223-237 (1992)

19. Nayfeh, A.H., Chimenti, D.E.: Ultrasonic wave reflection from liquid-coupled orthotropic plates with application to fibrous composites. J. Appl. Mech. 55, 863-870 (1988)

20. Zienkiewicz, O.C., Taylor, R.L.: The Finite Element Method: Solid Mechanics, vol. 2. Butterworth-heinemann, Oxford (2000)
21. Mead, D.: A general theory of harmonic wave propagation in linear periodic systems with multiple coupling. J. Sounds Vib. 27, 235260 (1973)

22. Langley, R.: A note on the force boundary conditions for twodimensional periodic structures with corner freedoms. J. Sounds Vib. 167, 377-381 (1993)

23. Mace, B.R., Duhamel, D., Brennan, M.J., Hinke, L.: Finite element prediction of wave motion in structural waveguides. J. Acoust. Soc. Am. 117(5), 2835-2843 (2005)

24. Waki, Y., Mace, B., Brennan, M.: Numerical issues concerning the wave and finite element method for free and forced vibration of waveguides. J. Sounds Vib. 327(1-2), 92-108 (2009)

25. Mencik, J.-M., Ichchou, M.: Multi-mode propagation and diffusion in structures through finite elements. Eur. J. Mech. A 24(5), 877898 (2005)

26. Chronopoulos, D., Troclet, B., Bareille, O., Ichchou, M.: Modeling the response of composite panels by a dynamic stiffness approach. Compos. Struct. 96, 111-120 (2013)

27. Manconi, E., Mace, B.: Modelling wave propagation in twodimensional structures using a wave/finite element technique. ISVR Technical Memorandum, (2007)

28. Chronopoulos, D., Troclet, B., Ichchou, M., Laine, J.: A unified approach for the broadband vibroacoustic response of composite shells. Compos. B 43(4), 1837-1846 (2012)

29. Apalowo, R., Chronopoulos, D., Ichchou, M., Essa, Y., Martin De La Escalera, F.: The impact of temperature on wave interaction with damage in composite structures. Proc. Inst. Mech. Eng. C 231(16), 3042-3056 (2017)

30. Apalowo, R., Ampatzidis, T., Chronopoulos, D., Ichchou, M., Essa, Y., De La Escalera, F.: Prediction of temperature dependent wave dispersion and interaction properties in composite structures. In: Proceedings of the 24th International Congress on Sound and Vibration, 23-27 July 2017, London, (2017)

31. Chronopoulos, D., Droz, C., Apalowo, R., Ichchou, M., Yan, W.: Accurate structural identification for layered composite structures, through a wave and finite element scheme. Compos. Struct. 182 566-578 (2017)

32. Renno, J.M., Mace, B.R.: Calculation of reflection and transmission coefficients of joints using a hybrid finite element/wave and finite element approach. J. Sound Vib. 332(9), 2149-2164 (2013)

33. Cook, R.: Concepts and Applications of Finite Element Analysis, 2nd edn. Wiley, New York (1981)

34. ANSYS, ANSYS 14.0 User's Help, (2014)

35. Talreja, R., Singh, C.: Damage and Failure of Composite Materials, Damage and Failure of Composite Materials. Cambridge University Press, Cambridge (2012)

36. Nag, A., Mahapatra, D., Gopalakrishnan, S., Sankar, T.: A spectral finite element with embedded delamination for modeling of wave scattering in composite beams. Compos. Sci. Technol. 63(15), 2187-2200 (2003)

37. Petyt, M.: Introduction to Finite Element Vibration Analysis. Cambridge University Press, Cambridge (2010)

38. Cremer, L., Heckel, M., Petersson, B.: Structure Borne Sound: Structural Vibrations and Sound Radiation at Audio Frequencies. Springer, New York (2005)

39. Lowe, M., Cawley, P., Kao, J., Diligent, O.: The low frequency reflection characteristics of the fundamental antisymmetric lamb wave a 0 from a rectangular notch in a plate. J. Acou. Soc. Am. 112(6), 2612-2622 (2002) 